\title{
Late Twenty-First-Century Changes in the Midlatitude Atmospheric Circulation in the CESM Large Ensemble ${ }^{\mathscr{A}}$
}

\author{
Y. PEINGS \\ Department of Earth System Science, University of California, Irvine, Irvine, California \\ J. CATTIAUX \\ Centre National de Recherches Météorologiques, UMR 3589 CNRS/Météo-France, Toulouse, France \\ S. VAVRUS \\ Center for Climatic Research, University of Wisconsin-Madison, Madison, Wisconsin \\ GUDRUN MAGNUSDOTTIR \\ Department of Earth System Science, University of California, Irvine, Irvine, California
}

(Manuscript received 27 April 2016, in final form 22 February 2017)

\begin{abstract}
Projected changes in the midlatitude atmospheric circulation at the end of the twenty-first century are investigated using coupled ocean-atmosphere simulations from the Community Earth System Model Large Ensemble (CESM-LENS). Different metrics are used to describe the response of the midlatitude atmospheric dynamics in 40 ensemble members covering the 1920-2100 period. Contrasted responses are identified depending on the season and longitudinal sector that are considered. In winter, a slowdown of the zonal flow and an increase in waviness is found over North America, while the European sector exhibits a reinforced westerly flow and decreased waviness. Extreme temperature events in midlatitudes are more sensitive to thermodynamical than dynamical changes, and a general decrease in the intensity of wintertime cold spells is found. Analyses of individual ensemble members reveal a large spread in circulation changes due to internal variability. Causes for this spread are found to be tied to the Arctic amplification in the Pacific-North American sector and to the polar stratosphere in the North Atlantic. A competition mechanism is also discussed between the midlatitude response to polar versus tropical changes. While the upper-tropospheric tropical warming pushes the jet stream poleward, in winter, Arctic amplification and the weaker polar vortex exert an opposite effect. This competition results in a narrowing of the jet path in the midlatitudes, leading to decreased/unchanged waviness/blockings. This interpretation somewhat reconciles conflicting results between the hypothesized effect of Arctic amplification and projected changes in midlatitude flow characteristics. This study also illustrates that further understanding of regional processes is critical for anticipating changes in the midlatitude dynamics.
\end{abstract}

\section{Introduction}

Anticipating possible changes in the midlatitude atmospheric dynamics under climate change is crucial for predicting their impacts on surface climate variability.

Supplemental information related to this paper is available at the Journals Online website: http://dx.doi.org/10.1175/ JCLI-D-16-0340.s1.

Corresponding author: Yannick Peings, ypeings@uci.edu
Extensive research on the subject in the last decade has identified several key mechanisms that may influence future changes in the midlatitude atmospheric circulation. One prominent signal of climate change is the larger surface warming in the northern high latitudes compared to other regions, a process known as Arctic amplification (AA; e.g., Holland and Bitz 2003; Kay et al. 2012). Because of AA, the Arctic warms more rapidly than other regions of Earth's surface, as identified in recent observations (Screen and Simmonds 2010; Perlwitz et al. 2015) and projections from fully coupled 
global climate models (GCMs) (e.g., Barnes and Polvani 2015). This asymmetry results in a decrease of the meridional temperature gradient at the surface, one of the key ingredients controlling the zonal flow and baroclinic instability in midlatitudes (e.g., Hall et al. 2014).

Recent studies have hypothesized that increasing AA would result in an increase in frequency of extreme events in midlatitudes, such as warm/cold spells, extreme snowfall, and floods (e.g., Francis and Vavrus 2012, hereafter FV12, 2015; Liu et al. 2012; Screen 2013). The mechanism, detailed in FV12, involves a chain of events (Overland et al. 2015), some of which are less certain than others. The FV12 mechanism can be decomposed into five parts: 1) AA induces higher geopotential heights at the pole; 2) westerly winds are weakened in the midlatitudes owing to thermal wind adjustment; 3) in a weaker westerly flow, the jet stream waviness increases, leading to higher occurrence of blocking events; 4) the increase in waviness/blocking results in more persistent weather patterns; and 5) more persistent weather patterns increase the likelihood of extreme weather events in the midlatitudes. The FV12 hypothesis has stimulated numerous studies on the matter [see reviews by Walsh (2014), Cohen et al. (2014), and Vihma (2014)] and led to the definition of new metrics to characterize the waviness of the midlatitude flow (Barnes 2013; Screen and Simmonds 2013; Chen et al. 2015). To this date, the FV12 mechanism remains highly debated and uncertain (Wallace et al. 2014; Barnes and Screen 2015). The shortness of the observational record and the chaotic nature of midlatitude atmospheric flow limit the identification of any AA-related midlatitude change in recent years (Barnes 2013; Screen and Simmonds 2013; Overland et al. 2015). Moreover, some studies offer an opposite view regarding the influence of AA on extreme weather events, by linking AA to a decrease in midlatitude surface temperature variability (Screen 2014; Screen et al. 2015; Schneider et al. 2015; Blackport and Kushner 2016; Ayarzagüena and Screen 2016) and a decrease in the occurrence of atmospheric blockings (Hassanzadeh et al. 2014; Hassanzadeh and Kuang 2015; Kennedy et al. 2016), challenging the reality of the FV12 mechanism.

Even under the hypothesis that AA actually leads to an increase in extreme weather events, it is not guaranteed that this effect dominates changes in the midlatitude climate at the end of the twenty-first century [as stated in Barnes and Screen (2015), "it can" does not imply that "it has" or "it will"]. In fact, AA is not the only process that could potentially modify the midlatitude atmospheric dynamics under climate change. The expansion of the Hadley cells and the large warming of the upper troposphere in the tropics, or upper-level tropical warming (UTW), are also large and robust signatures of climate change (Manabe and Wetherald 1975). UTW increases the meridional temperature gradient in the upper troposphere, which through thermal wind balance promotes a stronger zonal flow and a poleward shift of the jet stream (i.e., an opposite response to AA as hypothesized by FV12 and other studies). The future evolution of midlatitude atmospheric dynamics depends on both the upper- and lower-tropospheric temperature gradients (Held 1993) but with differences depending on the region and/or season (Butler et al. 2010; Harvey et al. 2013; Manzini et al. 2014). Of primary interest is the response in terms of the large-scale atmospheric modes of variability such as the northern annular mode (NAM; Thompson and Wallace 1998). Under increasing radiative forcing, the latest Coupled Model Intercomparison Project (CMIP5) simulations project a slight shift in the phase of the wintertime midtroposphere NAM toward more negative values that is partially related to AA and sea ice loss (Cattiaux and Cassou 2013). This negative NAM response in winter is supported by modeling studies that have investigated the atmospheric response to large Arctic sea ice reduction (e.g., Peings and Magnusdottir 2014; Deser et al. 2015). However, detailed analyses of smaller-scale midlatitude circulation metrics reveal the large spread in projected changes among GCMs and the lack of a consistent signal that directly supports the FV12 mechanism (Barnes and Polvani 2015). While in other seasons, there is good agreement for a poleward shift of the Northern Hemisphere $(\mathrm{NH})$ jet stream location in climate change projections of CMIP5 models, the winter season shows greater spread between the models (Simpson et al. 2014; Barnes and Polvani 2015). This also applies to the response of the polar stratospheric temperature (PST) (Manzini et al. 2014; Cattiaux et al. 2016), which is known to influence the midlatitude dynamics through stratosphere-troposphere coupling (e.g., Kidston et al. 2015). There is therefore a need for understanding the origins of intermodel spread in the midlatitude circulation, especially in winter when the zonal flow is the most active. One goal of this study is to explore how the changes in midlatitude circulation metrics may relate to the large-scale signatures of climate change such as AA, UTW, or PST at the end of the twenty-first century.

CMIP5 models vary a great deal in climate sensitivity and in amplitude of feedback mechanisms related to sea ice, clouds, and so forth (e.g., Andrews et al. 2012). Consequently, they exhibit different amplitudes of AA and UTW at the end of the twenty-first century, 


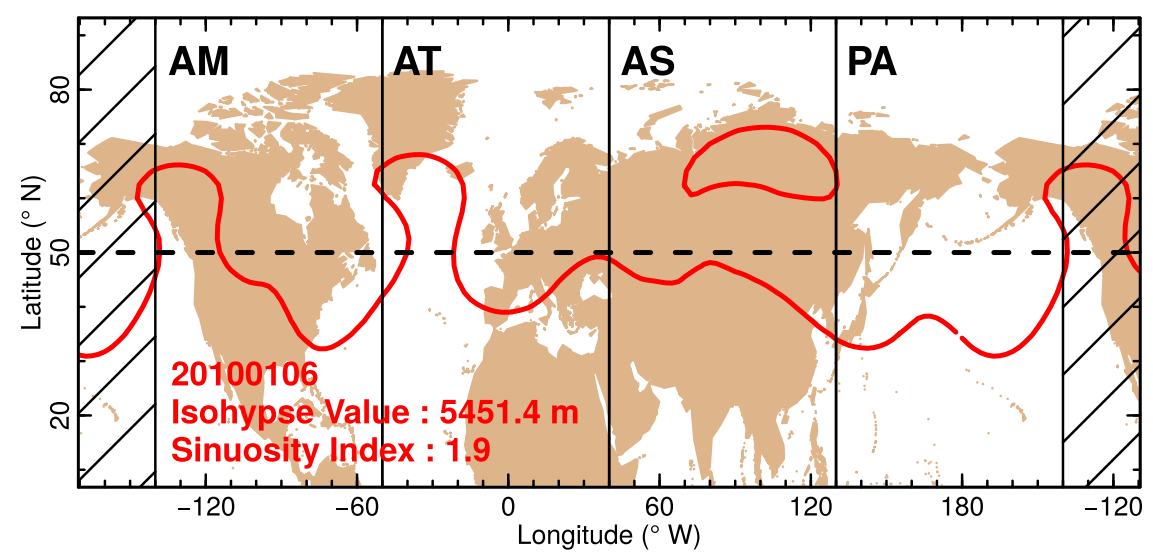

FIG. 1. Example of a daily isohypse isolated to compute the sinuosity index and spatial domain covered by each longitudinal sector (AM, AT, AS, and PA).

allowing one to assess how the dispersions in AA and UTW affect the dispersion in midlatitude circulation changes. Such an analysis is made in a complementary study that specifically focuses on one measure of the midlatitude atmospheric dynamics, which is the sinuosity or waviness of the flow (Cattiaux et al. 2016). Analyzing CMIP5 simulations presents the advantage of including consideration of model uncertainties, but it is limited by the small number of ensemble members available for each model ( $\leq 10$; Taylor et al. 2012). Internal climate variability is large, especially in the extratropics, and recent findings advocate for using larger ensembles of simulations when attempting to identify and attribute signals of climate change (Deser et al. 2014). For this purpose, the recent Community Earth System Model Large Ensemble project (CESMLENS; Kay et al. 2015) was conducted to provide a large ensemble of climate simulations for the twentieth and twenty-first centuries. This set of simulations enables investigating the climate response to increasing anthropogenic forcing without (or with greatly reduced) uncertainty related to internal climate variability. The present study explores how the mean flow and various metrics of the midlatitude atmospheric dynamics are modified in CESM-LENS at the end of the twenty-first century, under the representation concentration pathway $8.5 \mathrm{~W} \mathrm{~m}^{-2}$ (RCP8.5) scenario of increasing anthropogenic emissions. By using a single model, compared to Cattiaux et al. (2016), we neglect model uncertainties here. However, using a large ensemble of simulations from one single model allows us to explore different issues. In addition to effectively isolating the forced response, CESM-LENS also highlights the role of internal variability and of large-scale climate change signals in the spread of midlatitude circulation changes.
The paper is organized as follows. Section 2 describes the CESM-LENS set of simulations and defines the different metrics that we use. Section 3 presents results based on both the ensemble mean of the simulations and the intermember spread. Finally, section 4 concludes by summarizing and discussing our findings in a broader perspective.

\section{Methodology}

\section{a. Description of the CESM-LENS simulations}

CESM-LENS consists of 40 ensemble members (at the time of the study) integrated from 1920 to 2100 with the CESM1 fully coupled model. CESM1 includes atmosphere, ocean, land, and sea ice component models (Hurrell et al. 2013). The atmospheric model is version 5 of the Community Atmospheric Model (CAM5), with 30 vertical levels and a horizontal resolution of $1^{\circ}$. Historical radiative forcing is imposed for the period 1920-2005, and RCP8.5 radiative forcing thereafter. An overview of the CESM-LENS project is presented in Kay et al. (2015). Projected changes from present day to the late twenty-first century are estimated by comparing two 30-yr periods: 2071-2100 and 1981-2010. A two-tailed Student's $t$ test is used to determine the significance of the ensemble mean response, from the respective standard deviation of the 40-member ensemble.

\section{b. Definition of metrics}

Three main metrics are used in this study to characterize the midlatitude circulation. They are computed over five domains (see Fig. 1): the whole NH, North Atlantic (AT), Asia (AS), North America (AM), and North Pacific (PA). The metrics are constructed as follows, using daily data. Note that all indices are normalized for each ensemble member relative to the 1920-80 
period. They are therefore expressed in standard deviation units.

- The zonal index $(\mathrm{ZON})$ is computed as the difference in mean 500-hPa geopotential height Z500 between the $20^{\circ}-50^{\circ}$ and $60^{\circ}-90^{\circ} \mathrm{N}$ latitudinal bands (Woollings 2008). ZON characterizes the change in the mean state of the $\mathrm{NH}$ atmosphere, as the redistribution of mass between the pole and the midlatitudes. A positive ZON corresponds to a reinforced westerly flow, and vice versa. Unlike local metrics such as the North Atlantic Oscillation (NAO; Hurrell and van Loon 1997) index, ZON consistently characterizes the midlatitude zonal flow independently of the sector and/or season. ZON is highly correlated with the NAM and the NAO in the North Atlantic sector. In CESM-LENS, over 1920-2100, the average intermember correlation between the wintertime NH ZON and the NAM [defined as the first empirical orthogonal function (EOF) of $\mathrm{Z} 500$ in the $\mathrm{NH}]$ is 0.70 . The correlation is 0.91 between the wintertime NA ZON and the NAO (defined as the first EOF of Z500 in the North Atlantic sector). The correlations remain quite stationary in time, although the NAM-ZON correlation slightly increases during the course of the twentyfirst century as the part of the NAM in Z500 variability increases in the simulations (from $\sim 30 \%$ to $\sim 40 \%$ along the course of the simulations; not shown).

- The sinuosity index (SIN) characterizes the trajectory, or "waviness," of the midlatitude flow by isolating and measuring the length of a particular 500-hPa isohypse (i.e., a line of equal Z500), which may be discontinuous (see the example in Fig. 1). For each day, the value of the selected isohypse precisely corresponds to the Z500 average over $30^{\circ}-70^{\circ} \mathrm{N}$. For example, if for a particular day the averaged $\mathrm{Z} 500$ in the $30^{\circ}-70^{\circ} \mathrm{N}$ latitudinal band is $5660 \mathrm{~m}$, the corresponding daily sinuosity will correspond to the length of the $5660 \mathrm{~m}$ isohypse. By doing so, we ensure that we constantly describe the atmospheric flow at the same latitude (approximately $50^{\circ} \mathrm{N}$ ), which avoids potential pitfalls related to the seasonal cycle or the thermal rise of the 500-hPa surface due to global warming (Barnes 2013). The length of the isohypse is normalized by the length of the $50^{\circ} \mathrm{N}$ latitude circle, with a sinuosity of 1 corresponding to a purely zonal flow (SIN is nondimensional). More details on the SIN index are given in Cattiaux et al. (2016). Note that other diagnostics also characterize waviness of the midlatitude atmospheric flow, such as finite-amplitude wave activity (e.g., Chen et al. 2015).

- The blocking index (BLO) is the one-dimensional (1D) blocking index from Tibaldi and Molteni (1990).
Briefly, this index is computed from daily Z500 by identifying reversals of geopotential height gradient along the longitude. BLO describes blocking situations in the midlatitude atmosphere that occur on the intraseasonal time scale and are often associated with extreme weather events.

In summary, each of the three metrics provides different information on the atmospheric flow. $\mathrm{ZON}$ is a large-scale metric that measures the strength of the zonal mean flow. BLO is a binary index ( 0 or 1 values according to the presence or absence of blocking) that provides information on the presence of blocking patterns and therefore on the presence of meridional meandering of the flow. SIN locates in between the two metrics in the sense that, similar to BLO, it captures synoptic events, but similar to $\mathrm{ZON}$ it also depends on the zonal mean state of the flow (this is illustrated in section 3a).

In addition to these three large-scale metrics, we also measure the transient eddy activity in storm tracks as the monthly standard deviation of the 2-6-day bandpassfiltered daily Z500. Changes in extreme temperature are also investigated, defining warm spells as events with a daily maximum 2-m temperature above the 90th percentile of the daily temperature distribution for at least 6 consecutive days. Cold spells are defined as events with a daily minimum $2-\mathrm{m}$ temperature below the 10 th percentile of the daily temperature distribution for at least 6 consecutive days. For the two 1981-2010 and 2071-2100 periods, the 90th- and 10th-percentile thresholds are different. Indeed, they are taken as the 90th and 10th percentiles of the daily temperature distribution of the corresponding period, meaning that future warm/cold spells are considered extremes relatively to the future climate. Note that if one were to use the same threshold to define cold spells in both the present and future period, cold spells in winter would almost be eliminated owing to the large global warming signal in CESMLENS (Figs. S1a-c in the online supplemental material). Similarly, summertime extreme warm days from the present period become the norm in the future period, leading to a great increase in frequency/duration of warm spells if the same 90th-percentile threshold is used for both periods (Figs. S1d-f). By differentiating our definition of present versus future extreme events, we investigate how characteristics of extreme events from the future (in regard to the future climatological state) compare to those from the present (in other words our results illustrate changes in variance and higher moments, rather than in mean, of the daily temperature). Three characteristics of extreme warm/cold spells are analyzed: frequency (in \% of seasonal days), intensity 
a) BLO vs ZON - NH - JFM

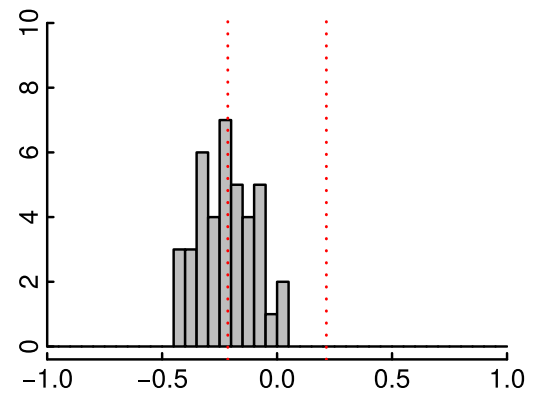

d) BLO vs ZON - NH - JAS

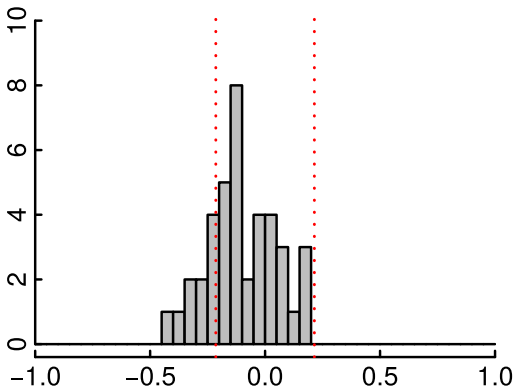

b) SIN vs ZON - NH - JFM

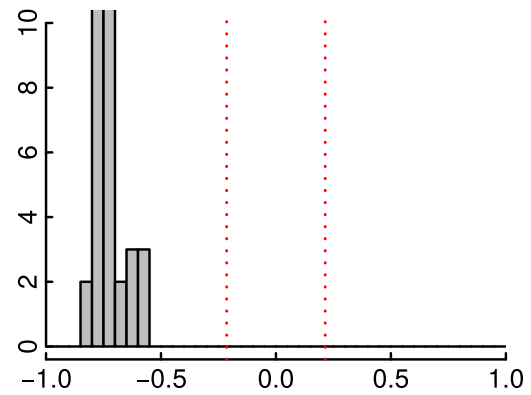

e) SIN vs ZON - NH - JAS

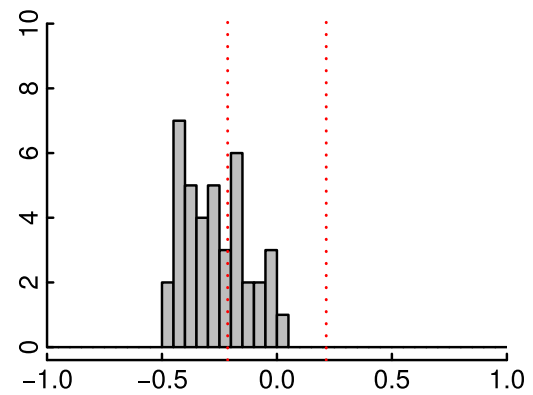

c) SIN vs BLO - NH - JFM

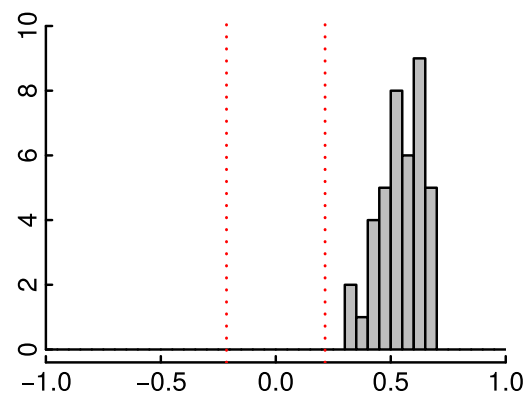

f) SIN vs BLO - NH - JAS

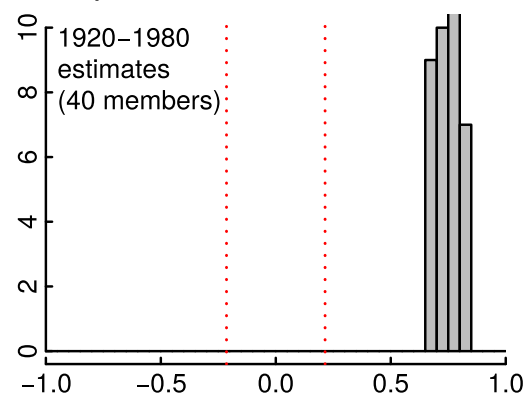

FIG. 2. The 40-member distribution of interannual correlations over 1920-80 between (a) BLO vs ZON, (b) SIN vs ZON, and (c) SIN vs BLO for the winter season (JFM) in average over the NH. (d)-(f) As in (a)-(c), but for summer (JAS). Red dashed vertical lines indicate the $95 \%$ significance level.

(expressed in ${ }^{\circ} \mathrm{C}$ as the mean anomaly during the event, relative to the daily climatology of the corresponding period), and duration (in days, by design equal to or greater than 6 days).

\section{Results}

\section{a. Interannual relationships between metrics}

Before investigating the changes in the circulation at the end of the twenty-first century, it is interesting to assess the relationships that exist among the different metrics in the CESM-LENS climatology. Figure 2 summarizes the interrelationships between the three $\mathrm{NH}$ circulation indices, ZON, BLO, and SIN, at the interannual time scale over the 1920-80 period. The 40-member distribution of correlations is given in winter [January-March (JFM)] and summer [July-September (JAS)].

In winter, large negative correlations are found between SIN and ZON (i.e., increase in sinuosity with a reduction in westerly wind), as well as positive correlations between SIN and BLO (i.e., increase in sinuosity associated with an increase in atmospheric blocking events). In contrast, the correlation between BLO and $\mathrm{ZON}$ is moderately negative with a larger intermember spread (correlation is only significant for half of ensemble members). These relationships are consistent with the third part of the FV12 mechanism (see introduction) and the idea that a weaker westerly flow is associated with increased waviness/blockings, although the link with atmospheric blocking is less pronounced.

In summer, the positive correlation between SIN and BLO is highly significant for all members. The negative relationships of SIN versus $\mathrm{ZON}$ and BLO versus $\mathrm{ZON}$ are less systematic, reflecting the weaker connection between synoptic-scale processes and large-scale westerlies in summer compared to winter.

\section{b. Mean changes at the end of the twenty-first century}

Figure 3 shows changes in various surface and circulation variables at the end of the twenty-first century, for the four seasons of the year [JFM, April-June (AMJ), JAS, and October-December (OND)]. Sea ice concentration strongly diminishes in all seasons (Fig. 3a), with a summertime sea ice-free Arctic occurring around 2070 (not shown). The loss of sea ice results in a large release of energy from the ocean into the atmosphere in fall and winter in the Arctic (Fig. 3b), as shown by the response of the total turbulent heat flux (sensible plus latent). Since heat flux anomalies are overly positive owing to the large global warming signal in the simulations, we removed the global mean of heat flux anomalies here. The same removal of global mean 
a) SIC
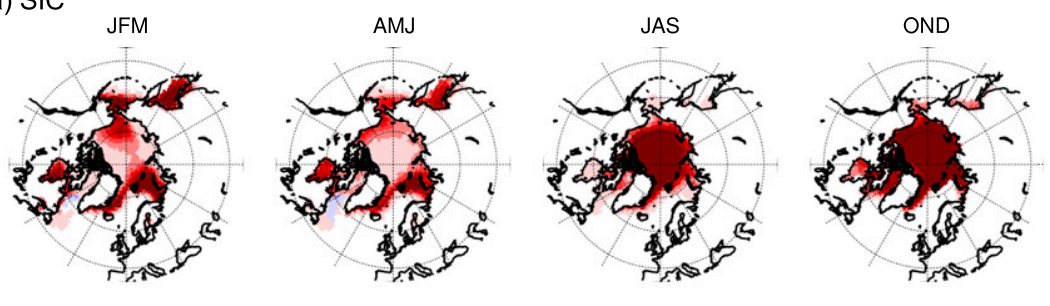

b) THFLX

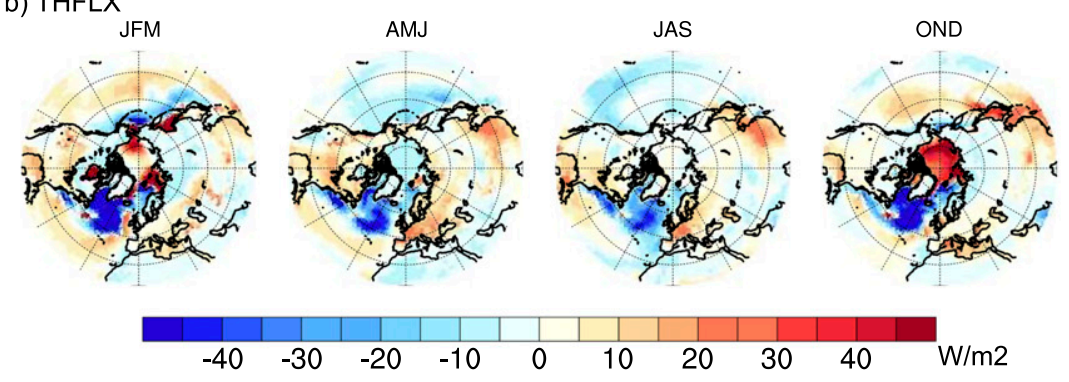

c) $\mathrm{T} 2 \mathrm{M}$

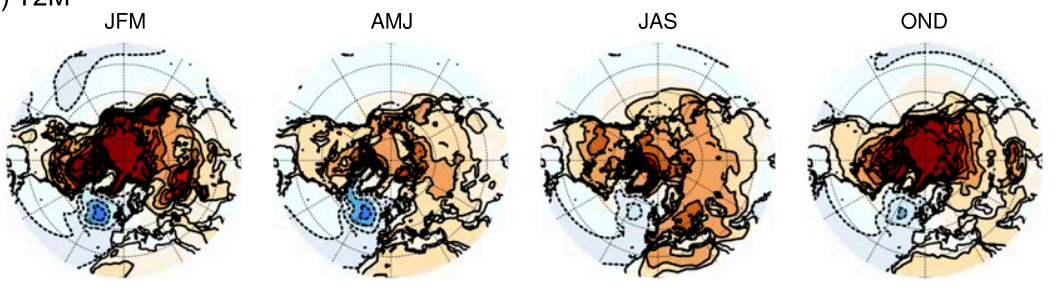

d) $Z 500$
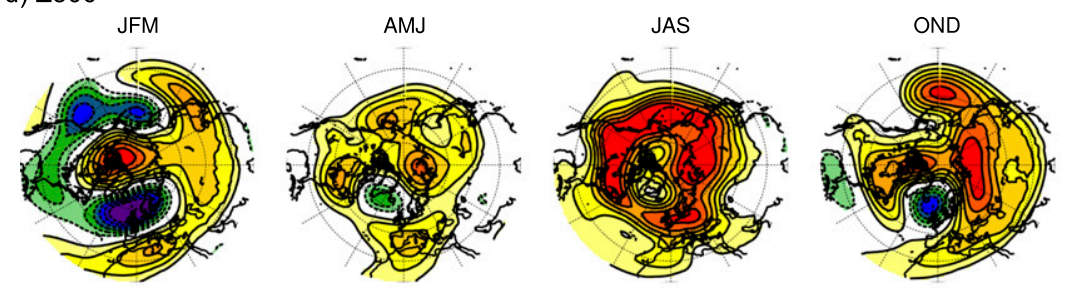

e) U10
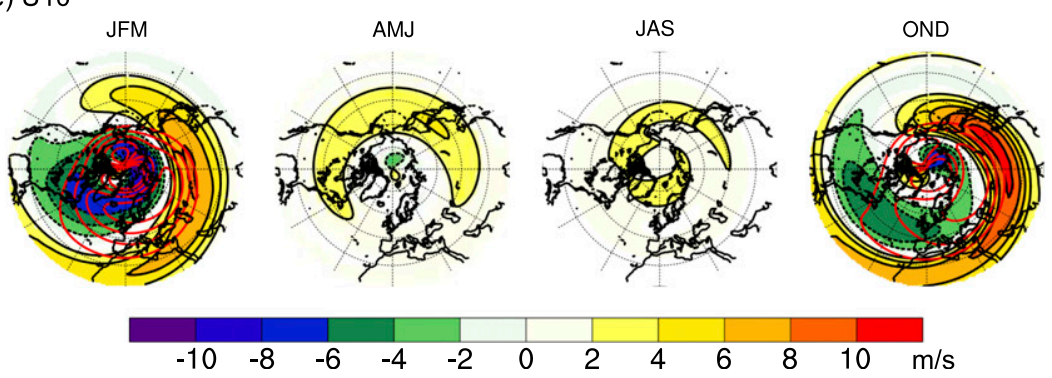

FIG. 3. Ensemble mean of seasonal changes between the 2071-2100 and the 1981-2010 periods for (a) sea ice concentration (\%), (b) turbulent heat flux (latent plus sensible; $\mathrm{W} \mathrm{m}{ }^{-2}$; global anomaly removed), (c) $2-\mathrm{m}$ temperature $\left({ }^{\circ} \mathrm{C}\right.$; global anomaly removed), (d) 500-hPa geopotential height (m; global anomaly removed), (e) 10-hPa zonal wind $\left(\mathrm{m} \mathrm{s}^{-1}\right)$ with red contours indicating the climatology over 1981-2010 (interval $10 \mathrm{~m} \mathrm{~s}^{-1}$ from 20 to $70 \mathrm{~m} \mathrm{~s}^{-1}$ ). Color shading indicates anomalies that are significant at the $95 \%$ confidence level. 
applies to the 2-m surface temperature (T2M; Fig. 3c) and to the 500-hPa geopotential height Z500 (Fig. 3d) for the same reason. Hence, anomalies in Figs. 3b-d highlight regional anomalies in the amplitude of the global change rather than the change itself. The Arctic and surrounding high-latitude continents warm more than other regions of the NH (Fig. 3c), as expected from the Arctic amplification signal largely forced by sea ice loss. The AA signal is less pronounced in spring and summer, when instead a large warming of the entire $\mathrm{NH}$ continents occurs that is promoted by decreased snow cover extent and reduced terrestrial heat capacity (Vavrus et al. 2017).

Despite the clear AA signal at the surface, in winter the pattern of the Z500 response (Fig. 3d) is more complicated than a simple negative NAM response as could be expected from the response to AA only (Deser et al. 2016). In JFM, it consists of a wave train with positive anomalies centered over the Beaufort Sea and spreading over Eurasia and negative anomalies from the North Pacific to the subpolar North Atlantic. Spatial correlation of the Z500 response with the NAM pattern, estimated as the first mode of an EOF performed on the concatenated 1920-2100 JFM Z500 anomalies from the 40 members, is -0.34 (moderate negative NAM). Changes in storm tracks (Fig. S2) reflect the Z500 anomalies, with an increase and southward shift of the transient eddy activity at the exit of the North Atlantic jet stream, over Europe and western Siberia. Conversely, the transient eddy activity is shifted poleward over the North Pacific, while decreasing over the Arctic. In summer, the Z500 anomalies are more closely related to the T2M change, with a large band of increasing Z500 over the warmer mid- to high-latitude continents. This is associated with a general decrease in storm-track activity in the midlatitudes (Fig. S2). The direct thermodynamical response to surface warming thus dominates in summer but not in winter when other processes related to large-scale dynamics influence the Z500 distribution (these processes are discussed later in the paper). Spring and fall anomalies represent a transition between the winter and summer anomalies.

Concerning the stratosphere, a large weakening of the polar vortex is found in winter, with weaker westerly winds at $10 \mathrm{hPa}$ around the Arctic and a shift of the polar vortex toward Eurasia (Fig. 3e). This signal is consistent with previous studies that have investigated the impact of AA and Arctic sea ice loss on the polar vortex (e.g., Peings and Magnusdottir 2014; Kim et al. 2014; Zhang et al. 2016). The weakening of the polar vortex over the American sector coincides with Z500 anomalies that correspond to a negative $\mathrm{ZON}$ in this region (Fig. $3 \mathrm{~d}$ and following results). Note that in section $3 \mathrm{e}$, we describe a strong connection between the strength of the polar vortex and the North Atlantic zonal index. This supports recent studies suggesting that stratosphere-troposphere coupling projects more strongly onto the NAO than the NAM (e.g., Hitchcock and Simpson 2014; Smith and Polvani 2014).

\section{c. Changes in metrics and impact on extremes of temperature}

We now investigate how the ZON, BLO, and SIN metrics are modified at the end of the twenty-first century (2071-2100 vs 1981-2010) at our latitude of interest, $50^{\circ} \mathrm{N}$. Figure 4 shows the annual cycle of change in the three metrics (unit is standard deviation since indices are normalized), with a confidence interval corresponding to the 40 -member spread $(75 \%$ of members are included in the error bars, or 30 out of 40 ). For SIN and BLO, relative anomalies in $\%$ are given on the right $y$ axis (green dashed line). Time series of each index are also shown on the right panels, for each domain and winter/summer.

\section{1) IN WINTER (JFM)}

We find no systematic change in NH ZON (Fig. 4a), but this signal represents a compensation between two opposite responses in the AT region (positive $\mathrm{ZON}$ anomalies) and in the $\mathrm{AM}$ region (negative $\mathrm{ZON}$ anomalies). This is consistent with Fig. $3 \mathrm{~d}$ and the longitudinal asymmetry in the Z500 response. In contrast, we find a decrease in SIN and BLO that represents a decrease in variability of the midlatitude circulation (Figs. 4b,c). This is especially pronounced over the AT and PA sectors. Meanwhile, BLO is unchanged and SIN is increased in the AM sector in association with the negative $\mathrm{ZON}$ anomaly. Based on these results, changes in the midlatitude flow as expected from the FV12 mechanism $(\mathrm{ZON}-/ \mathrm{SIN}+/ \mathrm{BLO}+)$ only occur over North America in CESM-LENS. This finding is detailed in Vavrus et al. (2017), who examine changes in zonal wind and sinuosity over the North American sector. In the other sectors, the response is opposite, with either a reinforced or unchanged westerly flow associated with decreased or unchanged waviness/blockings. The monthly SIN anomalies shown in Fig. 4 represent mean changes in SIN, thereby changes in quasistationary features of the circulation. To investigate changes in extreme sinuosity days (i.e., the tail of the SIN distribution), the distribution of SIN for 1981-2010 and 2071-2100 is shown in Fig. S3, for each longitudinal sector, in winter (Figs. S3a-e) and summer (Figs. S3f-j). In winter, the decrease in mean sinuosity over $\mathrm{NH}, \mathrm{AT}$, AS, and PA is associated with a narrowing of the SIN distribution and hence less variability and extreme 
a)

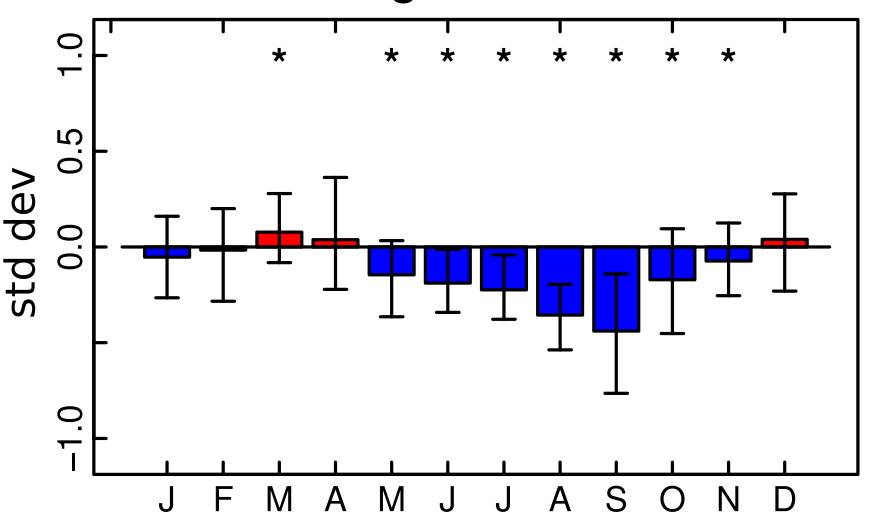

b)

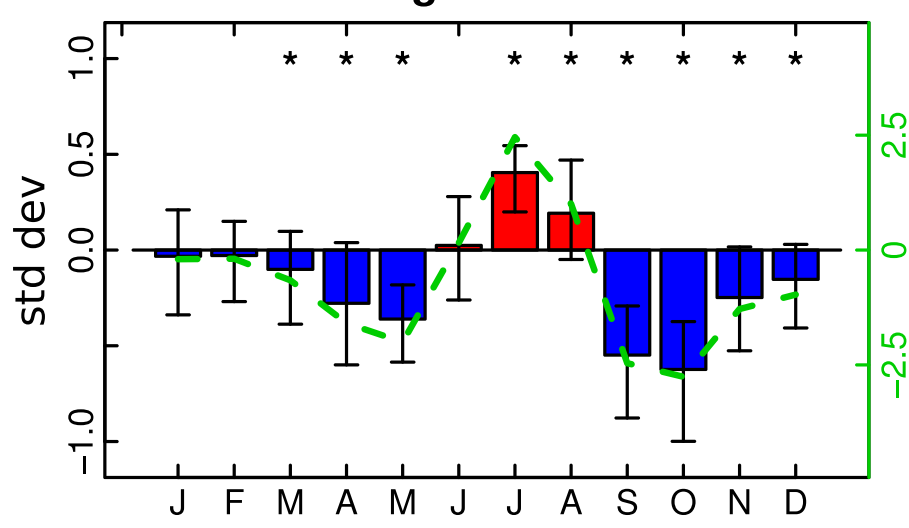

C)

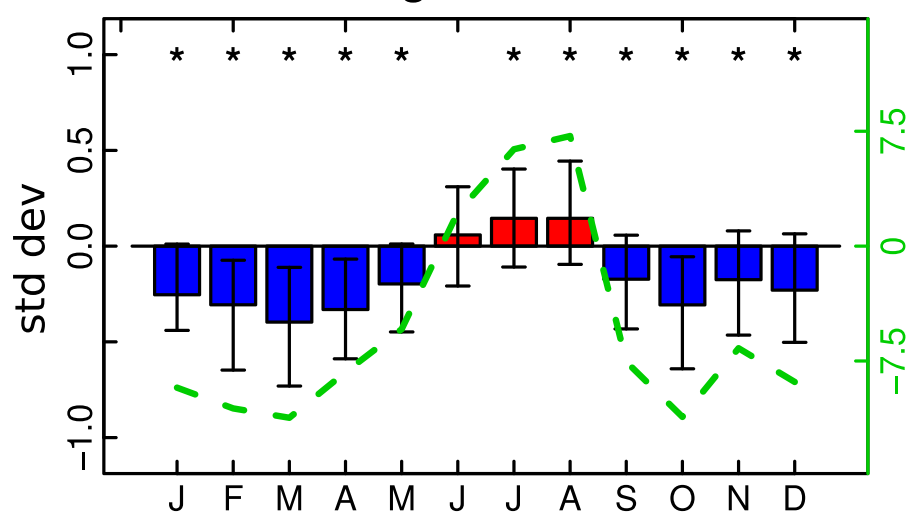

ZON - JFM
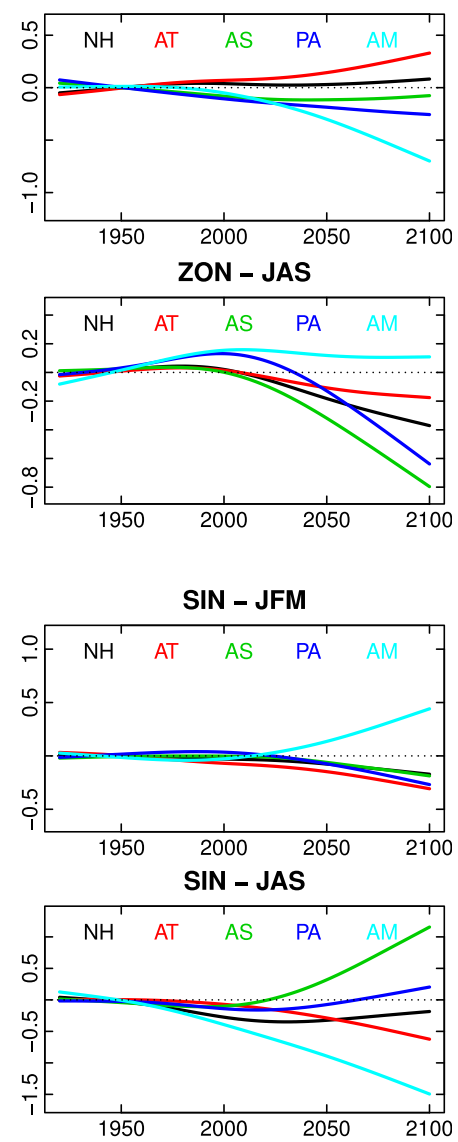

BLO - JFM
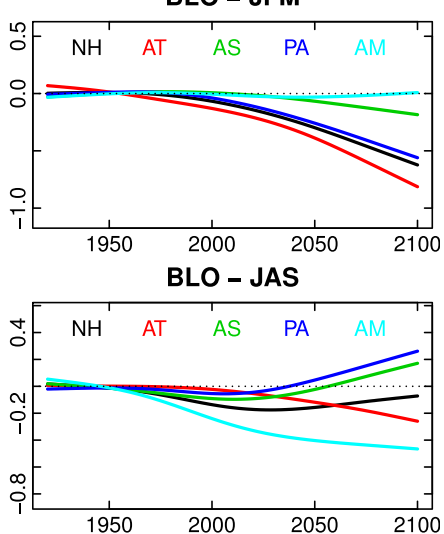

FIG. 4. Monthly changes (2071-2100 vs 1981-2010) in (a) ZON, (b) SIN, and (c) BLO, averaged over the entire NH. The colored bars show the 40-member ensemble mean, the whiskers indicate the intermember spread ( $75 \%$ of the members included, or 30 out of 40). The left axis unit is standard deviation and the right axis (green) gives the changes in \%. Stars indicate anomalies that are significant at the $95 \%$ significance level (Student's $t$ test). Corresponding 40-member ensemble mean time series of the metrics averaged over each sector (NH, AT, AS, PA, and $\mathrm{AM}$ ) in winter and summer are also shown to the right of each plot. 


\section{Cold spells - JFM}

\section{a) Frequency (\%)}

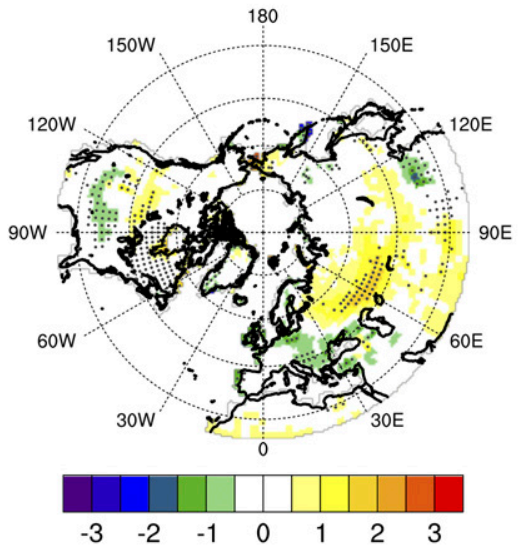

d) Frequency (\%)

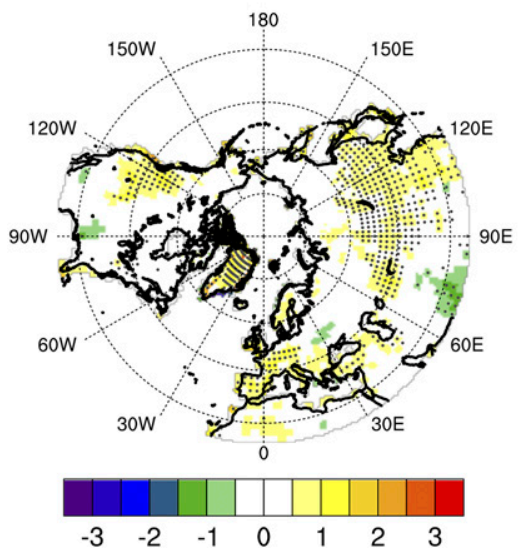

b) Intensity (C)

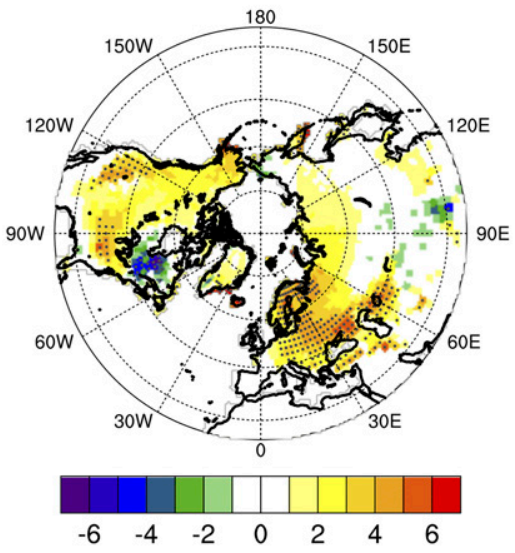

Warm spells - JAS

e) Intensity (C)

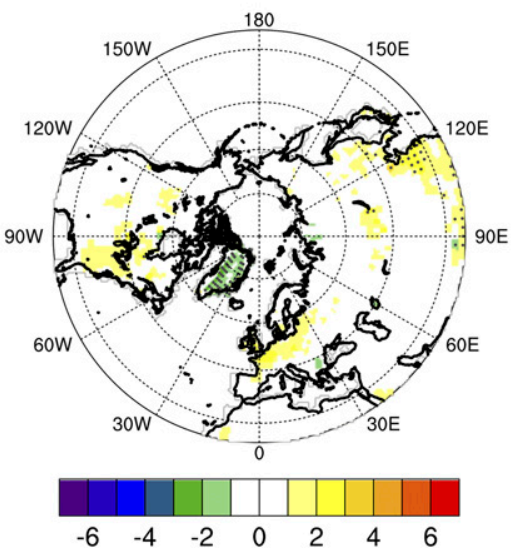

\section{c) Duration (day)}

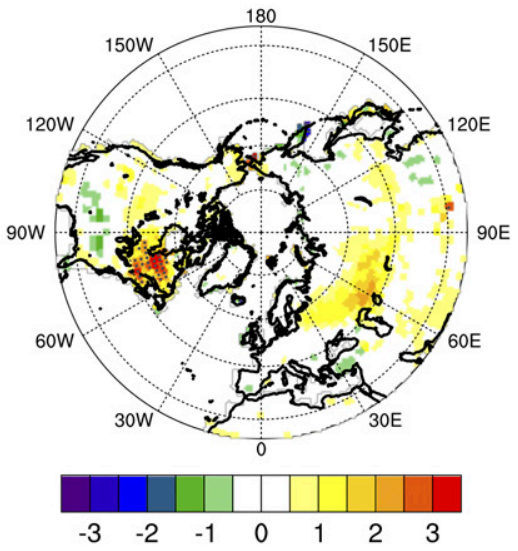

FIG. 5. Ensemble mean of JFM changes in cold spells between the 2071-2100 and the 1981-2010 periods in terms of (a) frequency (\%), (b) intensity $\left({ }^{\circ} \mathrm{C}\right.$ ), and (c) duration (days). (d)-(f) As in (a)-(c), but for warm spells in JAS. Stippling indicates anomalies that are significant at the $90 \%$ confidence level.

waviness. In contrast, the increase in mean sinuosity over AM is associated with a positive shift of the upper tail of the SIN distribution (i.e., more extreme waviness). In summer, the SIN distribution tends to broaden, especially over the AS and PA sector where an increase in mean SIN is found (i.e., more extreme waviness).

To estimate how the changes in dynamics impact the occurrence of extreme weather events, changes in cold spells are shown in Figs. 5a-c in terms of frequency, intensity, and duration. It is important to recall that for 2071-2100, cold spells are not defined relative to the present-day climate, but relative to the 2071-2100 distribution of temperature (see methodology in section $2 b$ and Fig. S1 for changes in cold/warm spells relative to the 1981-2010 distribution of temperature). Thus, the changes that are discussed here concern events that are considered cold/warm spells relatively to a warmer climate (we thus refer to them as "future cold/warm spells" in this section). Over Europe and North America, future cold spells are generally less frequent and less intense, except for eastern Canada where they increase both in intensity and duration (although their frequency remains unchanged). This local anomaly over eastern Canada is consistent with the presence of a ridge over the Arctic that advects polar air masses into this region (Fig. 3d), as well as with the increase in BLO/SIN in the AM sector (Figs. 4b,c). This ridge over the Arctic also impacts western Siberia, where future cold spells are more frequent, although they exhibit little or insignificant change in intensity and duration. Except for 
eastern Canada, the general decrease in intensity of future cold spells supports recent studies that project a decrease in daily temperature variability and less intense cold air outbreaks in midlatitudes in response to Arctic amplification (Screen 2014; Schneider et al. 2015; Ayarzagüena and Screen 2016). Although not demonstrated here, the mechanism is likely thermodynamical (i.e., driven by the weakening of the surface temperature gradient between the pole and the midlatitudes). In the projected future climatological state, polar air masses that are advected into midlatitudes during cold spells are relatively warmer than they are during the present period. In CESM-LENS, this thermodynamical effect of Arctic amplification seems to dominate dynamical changes everywhere into midlatitudes except over eastern Canada.

\section{2) IN SUMMER (JAS)}

We find a negative change in $\mathrm{ZON}$ in summer in all sectors except for AM (Fig. 4a), suggesting a general weakening of the westerly flow that is consistent with the pattern of Z500 anomalies in Fig. 3d. SIN and BLO exhibit more longitudinal asymmetry, with an increase in the AS/PA sectors and a decrease in the AM/AT sectors. Recall that our calculation of SIN emphasizes changes centered over the $50^{\circ} \mathrm{N}$ latitude, which can mask latitudinal asymmetry. By calculating SIN at each latitude band over North America, Vavrus et al. (2017) report large latitudinal variation in the changes in summertime SIN, which projects onto the dipole in zonal wind anomalies over this region (increased sinuosity with weaker westerlies, and conversely).

Changes in future warm spells are less pronounced than changes in future cold spells (Figs. 5d-f). Also they are not necessarily connected to dynamical changes. In particular, we find more frequent, more intense, and more persistent future warm spells over western Europe despite a decrease in SIN and BLO in the AT sector, which suggests the dominance of thermodynamical processes (e.g., local effects such as a soil moisture feedback can play a role here). On the other hand, the increase in intensity, frequency, and duration of future warm spells over Asia is consistent with the increase in SIN and BLO that is found in the AS sector.

\section{d. Uncertainty due to internal variability}

Figure 6 illustrates the amplitude of internal variability in terms of zonal mean NH changes for the three different metrics, by showing scatterplots of BLO versus ZON, SIN versus ZON, and SIN versus BLO anomalies in each individual ensemble member. Changes for winter and summer are shown in Figs. $6 \mathrm{a}-\mathrm{c}$ and $6 \mathrm{~d}-\mathrm{f}$, respectively. In accordance with the $75 \%$ model spread shown in Fig. 4 for monthly changes, the internal variability is very large, illustrating the danger of discussing future changes from a single or a small ensemble of realizations of one model. Even in terms of sign, there is little agreement among the ensemble members, except for BLO in winter and ZON in summer, which both show a decrease in most ensemble members.

As discussed in section 3c, dynamical changes implied by the FV12 mechanism (reduced westerlies and increased blocking/waviness) do not dominate future changes as simulated by CESM-LENS, except for the North American sector. However, SIN+ anomalies are mostly present in members that exhibit a $\mathrm{ZON}-$ anomaly (and vice versa; Fig. 6b) and in members with an increase or a small decrease in BLO (Fig. 6c). Except for BLO versus $\mathrm{ZON}$ in summer (Fig. 6d), all intermember correlations are statistically significant. Similar to interannual correlations (Fig. 2), this analysis supports part 3 of the FV12 mechanism (i.e., the amplitude of waviness/blockings is inversely correlated to the strength of the zonal flow). Now, a question we ask is whether the uncertainty in the response (i.e., the intermember spread) depends on the amplitude of Arctic amplification in each individual member or if it is related to other aspects of climate change. This question specifically addresses the main assumption of the FV12 mechanism (i.e., AA is the key driver of midlatitude dynamical changes and of the associated increase in extreme weather events). Even though this hypothesis is not supported by the mean changes of CESM-LENS, it is possible that the spread in AA explains some of the spread in the midlatitude changes. This is addressed in the following section.

\section{e. What controls the spread in the midlatitude dynamical changes?}

AA is only one component of climate change; its impacts on the midlatitude circulation depend on its interplay with the effect of other large-scale changes. In particular, the UTW is very pronounced at the end of the twenty-first century in CESM-LENS (Fig. 7a). Several studies have suggested that this signal increases the upper-level meridional temperature gradient and promotes a poleward shift of the polar front jet stream (e.g., Held 1993), which is opposite to the theoretical effect of AA (i.e., a decrease in the meridional temperature gradient near the surface). This competition between UTW and AA is key to anticipating changes in the midlatitude circulation, especially in winter. The present section aims to determine whether the intermember spread is related to the amplitude of "hot spot" signals of zonal temperature change in CESMLENS, including AA. 
a) BLO vs ZON - NH JFM

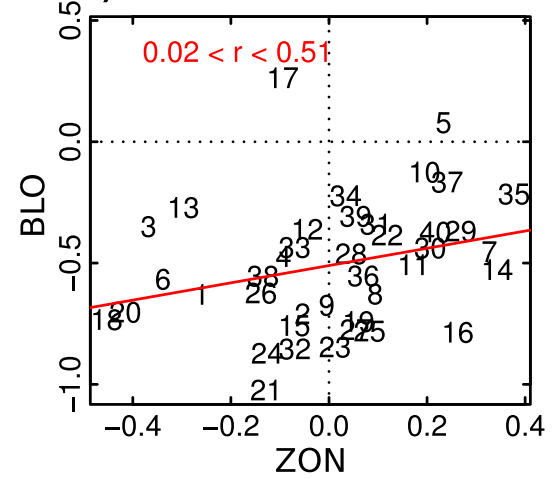

d) BLO vs ZON - NH JAS

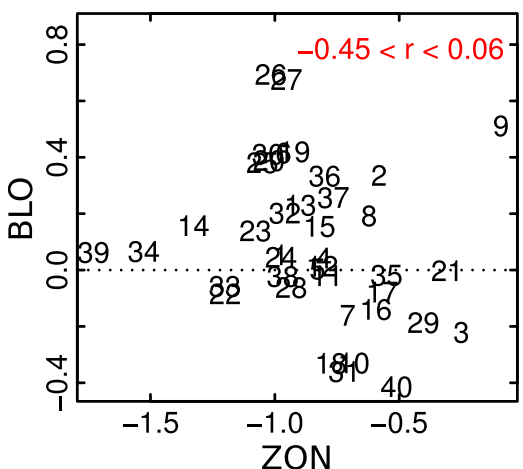

b) SIN vs ZON - NH JFM

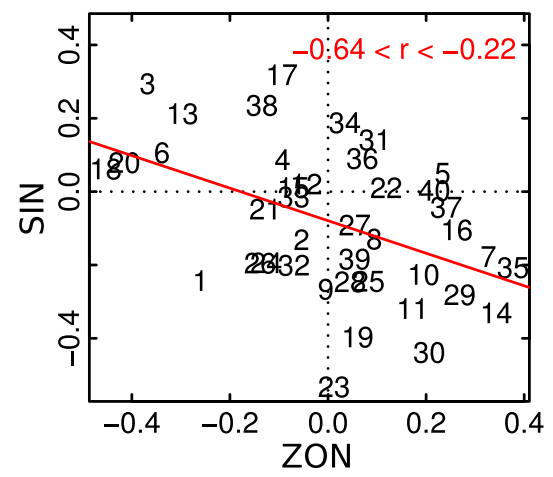

e) SIN vs ZON - NH JAS

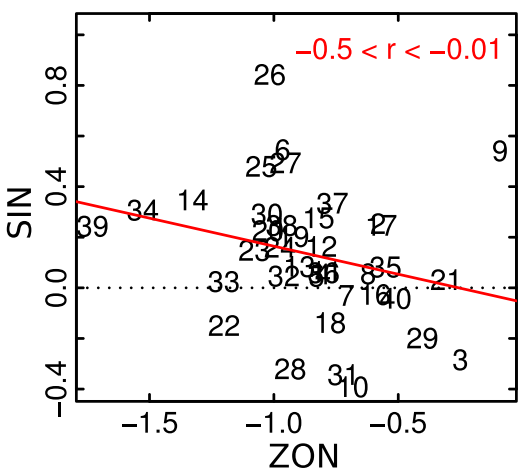

c) SIN vs BLO - NH JFM

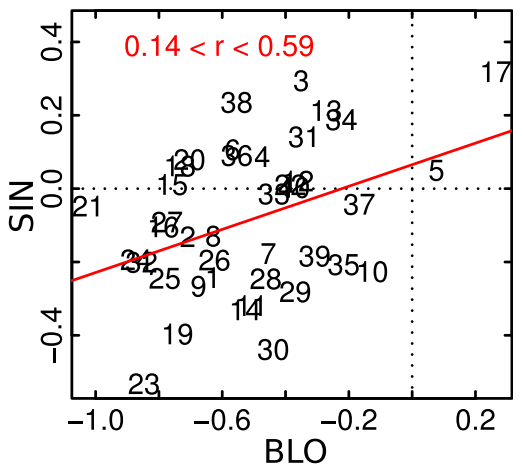

f) SIN vs BLO - NH JAS

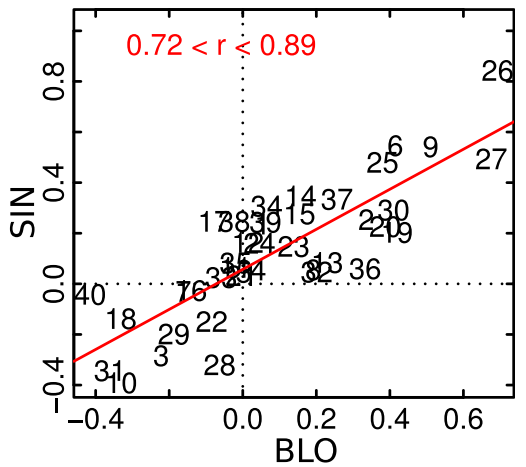

FIG. 6. The 40-member scatterplot of late twenty-first-century changes corresponding to the different metrics: (a) BLO vs ZON, (b) SIN vs ZON, and (c) SIN vs BLO, in winter (JFM) and in average over the NH. (d)-(f) As in (a)-(c), but for summer (JAS). Each member is indicated by a number; a linear regression line is plotted when the correlation is significant at the $95 \%$ confidence level (the confidence interval for $r$ is also given).

Figure 7 presents the relationships between changes in the wintertime metrics and five specific signals of zonal mean temperature change, in average over NH. Figures $7 \mathrm{~b}-\mathrm{f}$ respectively show some scatterplots between changes in $\mathrm{NH}$ $\mathrm{ZON}$ and changes in the upper-tropospheric temperature gradient (UPTG), the lower-tropospheric temperature gradient (LOTG), UTW, the surface polar warming or Arctic amplification (AA), and the temperature anomaly of the polar lower stratosphere (or PST). Figures $7 \mathrm{~g}-\mathrm{k}$ show a similar scatterplot using changes in SIN, while Figs. 71-p use changes in BLO. The different domains that are used to compute UPTG, LOTG, UTW, AA, and PST are shown in Fig. 7a. Their exact definition is given in Table 1. To assess regional dependences, Table 2 groups the correlations between the metrics and each temperature index for each individual sector. Correlations are shown as an interval of 95\% confidence level. Note that intercorrelations between UTW, AA, and PST changes are weak (Fig. S4), such that the amplitude of UTW, AA, and PST is unrelated among the ensemble members.

To discuss the most significant signals, large amplitude correlations (confidence interval above/below the
$0.3 /-0.3$ thresholds) are indicated in bold in Table 2 . Note that UTW is such a robust feature of climate change in CESM-LENS that it exhibits very little intermember variability (temperature anomalies vary only by a few tenths of a degree between ensemble members; see, e.g., the $x$ axis in Fig. 7d). Therefore we cannot really assess the dependence of changes in midlatitude dynamics to UTW in the present study. CMIP5 simulations are more appropriate for this task since they exhibit much more ensemble dispersion in this signal owing to different climate sensitivities and/or feedbacks (Cattiaux et al. 2016). We summarize the main findings from Fig. 7 and Table 2 below:

- ZON is significantly correlated with the upper-troposphere gradient of temperature (Fig. 7b), and thereby members with the largest increase in UPTG (tropics warming faster than the pole in the upper troposphere) are the members with larger positive $\mathrm{ZON}$ anomalies. This linkage is not due to UTW (Fig. 7d; but again there is too little variance in UTW to reach robust conclusions) but rather due to PST. Indeed, a larger decrease in $\mathrm{ZON}$ is associated 

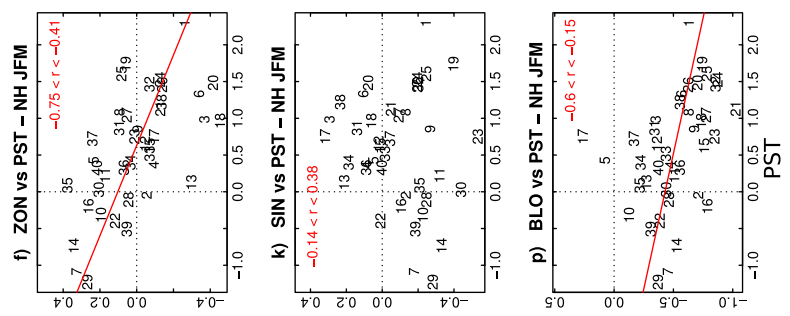

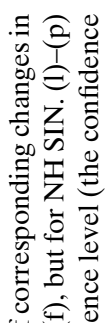
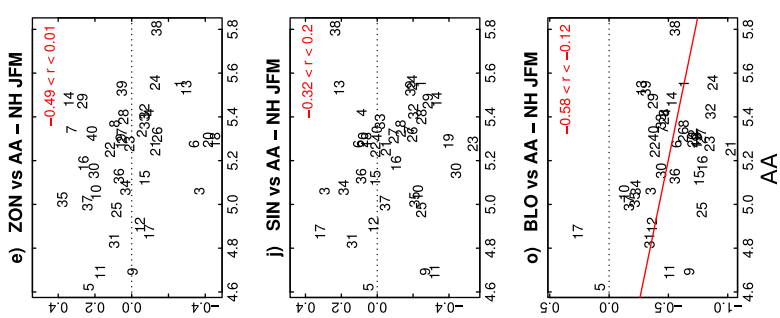

跣

응 잉

氖军学

त्

मे

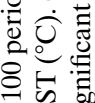
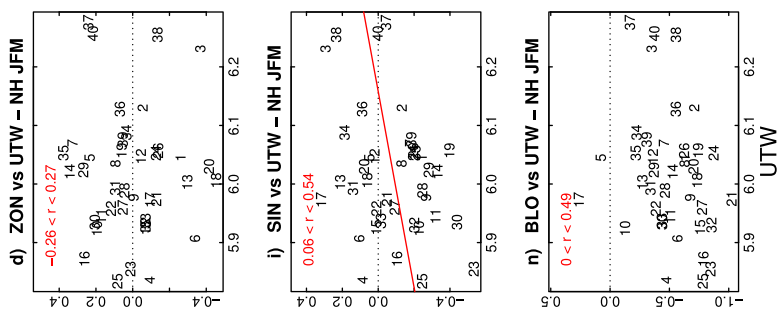

T.

동

공

可的

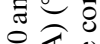

完安

요요
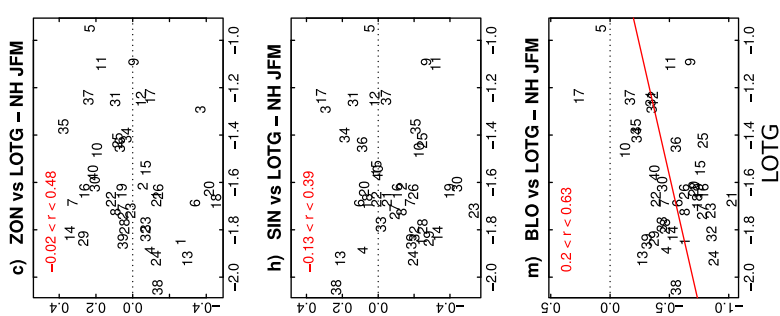

응

荺 :
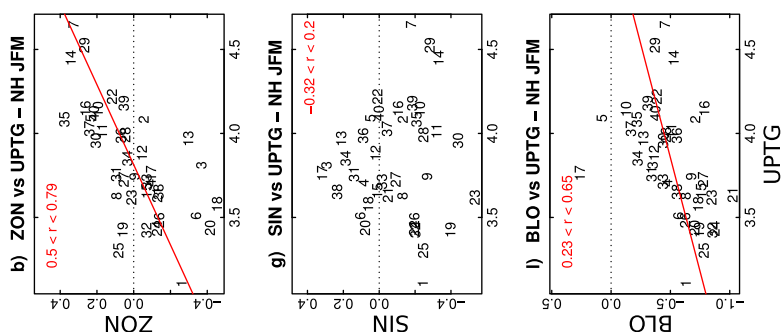

压

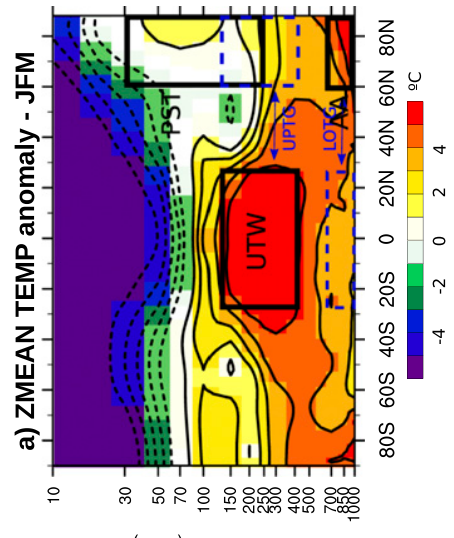

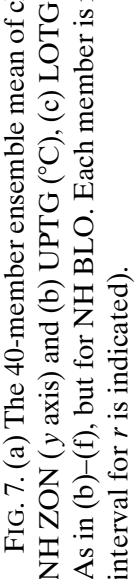


TABLE 1. Domain definition of zonal mean temperature indices used in Fig. 7.

\begin{tabular}{|c|c|c|c|c|c|}
\hline Temperature index & UPTG & LOTG & UTW & $\mathrm{AA}$ & PST \\
\hline Latitude range & $20^{\circ} \mathrm{S} / 20^{\circ} \mathrm{N}-60^{\circ} \mathrm{N} / 90^{\circ} \mathrm{N}$ & $20^{\circ} \mathrm{S} / 20^{\circ} \mathrm{N}-60^{\circ} \mathrm{N} / 90^{\circ} \mathrm{N}$ & $20^{\circ} \mathrm{S} / 20^{\circ} \mathrm{N}$ & $60^{\circ} \mathrm{N} / 90^{\circ} \mathrm{N}$ & $60^{\circ} \mathrm{N} / 90^{\circ} \mathrm{N}$ \\
\hline Vertical levels (hPa) & 400 to 150 & 1000 to 700 & 400 to 150 & 1000 to 700 & 250 to 30 \\
\hline
\end{tabular}

with warmer PST anomalies and hence a weaker polar vortex (Fig. 7f). ZON is only moderately correlated with AA (Fig. 7e) except over the PA/AM sector, where a larger AA is associated with larger negative $\mathrm{ZON}$ anomalies. In contrast, the connection to PST is only significant over the AT sector (Table 2), revealing a connection between changes in the lower polar stratosphere and NAO anomalies in the troposphere (Hitchcock and Simpson 2014; Smith and Polvani 2014). A high correlation between changes in PST and in the midlatitude atmospheric circulation has also been reported in the CMIP5 intermodel spread (Manzini et al. 2014; Cattiaux et al. 2016), supporting the finding that PST is strongly tied to the midlatitude circulation in late twenty-first-century RCP8.5 projections.

- Changes in SIN generally exhibit weaker linkages with the zonal temperature anomalies than $\mathrm{ZON}$ (Figs. $7 \mathrm{~g}-\mathrm{p}$ and Table 2). Yet interesting connections are found in individual subsectors (Table 2). In the PA sector, SIN increases with AA, in association with larger negative ZON anomalies. Over the Atlantic sector, similar to $\mathrm{ZON}$, the strongest connection is found with PST. BLO shows only moderate correlations, except for a link with PST in the PA sector (Table 2).

Overall, these findings illustrate the complexity of interpreting and anticipating future changes in midlatitude dynamics since the connection with large-scale features of climate change vary longitudinally. Our findings suggest that an effect of AA as expected from the FV12 mechanism is at work in the Pacific-North American sector, while the Atlantic sector has a larger connection with the lower polar stratosphere. This is further illustrated in Table 3, which gives the skill of different linear regression statistical models in predicting the intermember spread in ZON changes, using every possible combination of UTW, AA, and PST predictors. The largest skill is obtained using PST alone in the NH and AT sector ( $30 \%$ of explained variance), while AA + PST, UTW + AA, and AA alone provide the highest skills in the AS, PA, and AM sectors, respectively.

\section{$f$. Changes in the midlatitude jet stream due to polar versus tropical competing mechanisms}

As discussed in the last section, UTW exhibits little variance in CESM-LENS at the end of the twenty-first century so it has little impact on the spread of changes in midlatitude metrics. However, this does not mean that UTW does not have an influence on the ensemble mean response to climate change. To illustrate this and discuss the relative influence of polar changes compared to the influence of the Hadley cell expansion in the tropics, we are interested in the changes in the midlatitude (or eddy driven) jet streams on the hemispheric scale. The analysis is expanded to the Southern Hemisphere ( $\mathrm{SH}$ ) where AA is absent, such that comparing the NH to the $\mathrm{SH}$ highlights the influence of AA (and PST).

TABLE 2. Correlations between the ZON/BLO/SIN metrics and the temperature indices (see definitions in Table 1) in JFM, for each longitudinal sector (NH, AT, AS, PA, AM). Only correlations that are significant at the $95 \%$ confidence interval are shown, and the $95 \%$ confidence interval of correlations is given. Confidence interval of correlations that are above (below) the $0.3(-0.3)$ thresholds are in bold.

\begin{tabular}{|c|c|c|c|}
\hline & $\mathrm{ZON}$ & SIN & BLO \\
\hline \multicolumn{4}{|c|}{ UPTG } \\
\hline NH & $0.50 / 0.79$ & - & $0.23 / 0.65$ \\
\hline $\mathrm{AT}$ & $0.55 / 0.82$ & $-0.71 /-0.32$ & - \\
\hline AS & $0.08 / 0.55$ & - & - \\
\hline PA & - & - & $0.13 / 0.58$ \\
\hline $\mathrm{AM}$ & - & - & $0.05 / 0.53$ \\
\hline \multicolumn{4}{|c|}{ LOTG } \\
\hline NH & $-0.02 / 0.48$ & - & $0.20 / 0.63$ \\
\hline $\mathrm{AT}$ & - & - & $0.06 / 0.54$ \\
\hline AS & $0.04 / 0.53$ & - & $-0.55 /-0.08$ \\
\hline PA & $0.29 / 0.68$ & $-0.71 /-0.33$ & $-0.52 /-0.04$ \\
\hline $\mathrm{AM}$ & $0.42 / 0.76$ & $-0.53 /-0.05$ & - \\
\hline \multicolumn{4}{|c|}{ UTW } \\
\hline $\mathrm{NH}$ & - & $0.06 / 0.54$ & - \\
\hline $\mathrm{AT}$ & - & - & - \\
\hline AS & - & - & $0.09 / 0.56$ \\
\hline PA & - & $0.01 / 0.51$ & - \\
\hline $\mathrm{AM}$ & - & - & - \\
\hline \multicolumn{4}{|c|}{ AA } \\
\hline $\mathrm{NH}$ & $-0.50 /-0.01$ & - & $-0.58 /-0.12$ \\
\hline $\mathrm{AT}$ & - & - & $-0.50 /-0.01$ \\
\hline AS & $-0.54 /-0.06$ & - & $0.09 / 0.56$ \\
\hline PA & $-0.71 /-0.34$ & $0.3 / 0.72$ & $0.04 / 0.52$ \\
\hline $\mathrm{AM}$ & $-0.76 /-0.42$ & $0.06 / 0.54$ & - \\
\hline \multicolumn{4}{|c|}{ PST } \\
\hline $\mathrm{NH}$ & $-0.75 /-0.41$ & - & $-0.60 /-0.15$ \\
\hline $\mathrm{AT}$ & $-0.76 /-0.43$ & $0.30 / 0.69$ & - \\
\hline AS & - & - & - \\
\hline PA & - & $-0.63 /-0.19$ & $-0.73 /-0.37$ \\
\hline $\mathrm{AM}$ & - & - & $-0.56 /-0.09$ \\
\hline
\end{tabular}


TABLE 3. Percentage of variance explained by different linear regression models using combinations of JFM UTW, AA, and PST to predict intermember spread in JFM ZON changes in each sector. The skill of the statistical models is evaluated using a leave-one-out cross-validation method, and the adjusted variance is given (the power of the different models is corrected according to the number of predictors included). For each sector, the highest explained variance is in bold.

\begin{tabular}{|c|c|c|c|c|c|c|c|}
\hline $\mathrm{ZON}$ & UTW & AA & PST & $\mathrm{UTW}+\mathrm{AA}$ & UTW + PST & $\mathrm{AA}+\mathrm{PST}$ & $\mathrm{UTW}+\mathrm{AA}+\mathrm{PST}$ \\
\hline $\mathrm{NH}$ & 0 & 0 & 29 & 0 & 2 & 23 & 5 \\
\hline $\mathrm{AT}$ & 0 & 0 & 30 & 0 & 6 & 28 & 6 \\
\hline AS & 2 & 10 & 3 & 0 & 3 & 34 & 6 \\
\hline PA & 3 & 4 & 0 & 10 & 1 & 9 & 10 \\
\hline $\mathrm{AM}$ & 0 & 18 & 2 & 18 & 1 & 15 & 15 \\
\hline
\end{tabular}

Figure 8a depicts the climatological (1981-2010; contours) and anomalous (2071-2100 vs 1981-2010; shading) annual cycle of the 700-hPa zonal mean zonal wind (U700). This plot is similar to Fig. 6 in Deser et al. (2015), and it allows us to track monthly changes in the jet stream location/amplitude in the lower troposphere. In the SH, a persistent poleward shift of the jet stream is found all yearlong that is the signature of UTW in the absence of a strong polar amplification. Note that unlike the $\mathrm{NH}$, the stratosphere cools between 70 and $20 \mathrm{hPa}$ in the SH (Fig. 7a). This anomaly supplements with the effect of UTW in inducing a poleward shift of the jet in the $\mathrm{SH}$. In contrast, changes in the $\mathrm{NH}$ jet stream are not persistent throughout the year, with a poleward shift during the warm season that is mitigated in winter by negative wind anomalies in the high latitudes (Fig. 8a). This pattern represents a reinforcement and narrowing of the jet stream path, with positive anomalies around $50^{\circ} \mathrm{N}$ surrounded by negative anomalies in lower and higher latitudes. Maps of changes in U700 are shown in Fig. S5 to complement this zonally averaged picture with the regional distribution of the seasonal changes. In winter, the narrowing and reinforcement of the jet occurs mainly over Europe, at the exit of the North Atlantic eddy-driven jet, as well as in the North Pacific (tripole of U700 anomalies) where the linkage with AA has been shown to be important (section 3e). In other seasons, when AA is smaller and the midlatitude westerly flow is weaker, the westerlies decrease in the AS/PA sectors and are shifted poleward in the AM/AT sectors, consistent with summer changes in metrics that have been identified in section 3c. Figure S6 (pressure vs latitude cross section of the zonal mean zonal wind anomalies) reveals that the polar front jet stream is narrowed from the surface up to $300 \mathrm{hPa}$. Higher in the troposphere, the signal is dominated by positive westerly anomalies owing to a strengthened subtropical jet.

To highlight the role of AA in driving this pattern, Figs. $8 \mathrm{~b}-\mathrm{d}$ show the U700 anomalies, in winter only, for the 10 members with the lowest AA (Fig. 8b), the 10 members with the highest AA (Fig. 8c), and their difference (Fig. 8d). AA reinforces the northern part of the tripole anomaly (i.e., it pushes the jet southward). Figures $8 \mathrm{e}-\mathrm{g}$ present the same analyses but based on the amplitude of PST (warm vs cold PST). In line with the results from section 3e, PST is found to exert a larger influence than AA on the jet stream changes, with reinforced anomalies in the NH reflecting a narrower jet path when PST is warm. Of course, the question of causality is open here, since it is possible that PST responds to U700 changes through troposphere-stratosphere interactions, rather than forcing them (Kidston et al. 2015). This question is beyond the scope of the present study and it deserves further analyses to identify causality. The question of causality is also valid for UTW and AA, although they are more likely to drive the midtroposphere circulation than the opposite. Indeed, UTW and AA are typical signatures of climate change in GCMs that have been linked to other processes than changes in the troposphere dynamics (e.g., local feedbacks and low-frequency oceanic oscillations in the case of AA; Perlwitz et al. 2015).

The "squeezing" of the jet around $50^{\circ} \mathrm{N}$ is consistent with the previously identified decrease in BLO and SIN in winter (Fig. 4) since it prevents an increase in waviness of the flow that is confined to a narrower latitude band. This interpretation reconciles the FV12 mechanism with recent studies that found conflicting changes in midlatitude metrics (Barnes and Polvani 2015; Hassanzadeh and Kuang 2015). As hypothesized by FV12, AA indeed induces a southward shift of the jet stream. However, in future climate projections UTW counteracts this effect by pushing the jet in the opposite direction. Rather than a simple poleward or equatorward shift that would result from one effect overwhelming the other, the combination of AA and UTW on both sides of the jet reduces its meridional extent, hence the meridional meandering of the midlatitude flow.

\section{Discussion and conclusions}

CESM-LENS projects a large decrease in Arctic sea ice at the end of the twenty-first century, associated 
a) ZMEAN U700 anomalies - Annual cycle

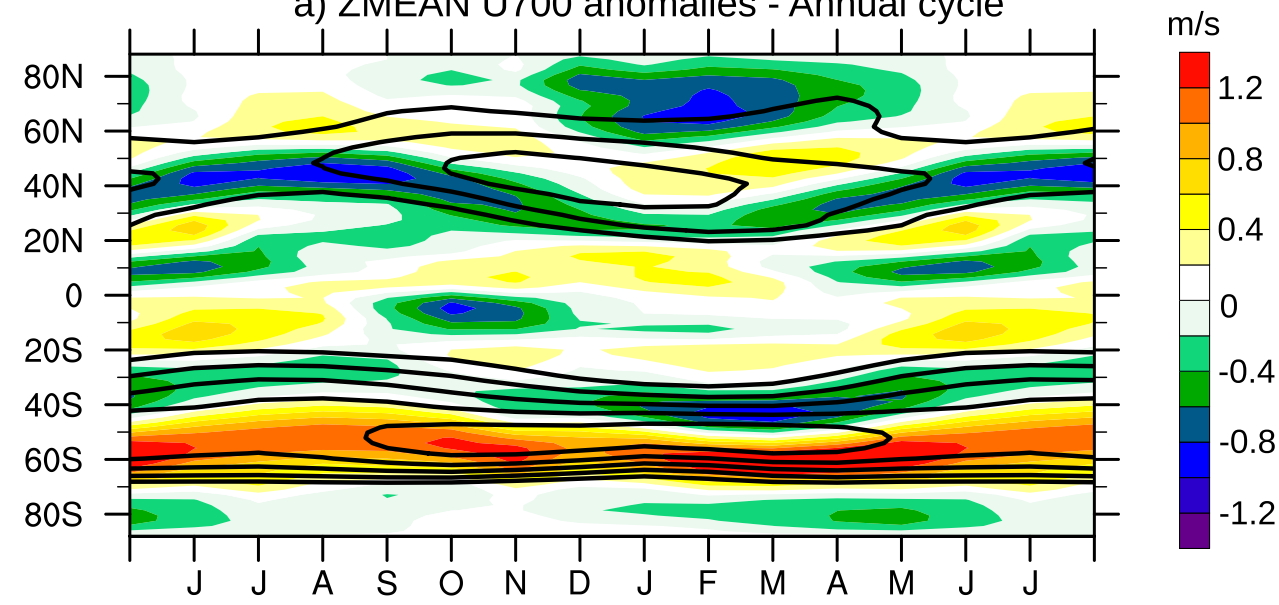

b) Low AA - JFM

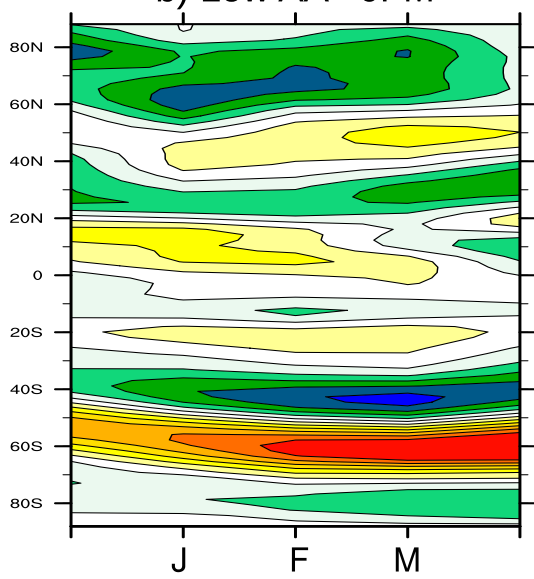

e) Cold PST - JFM

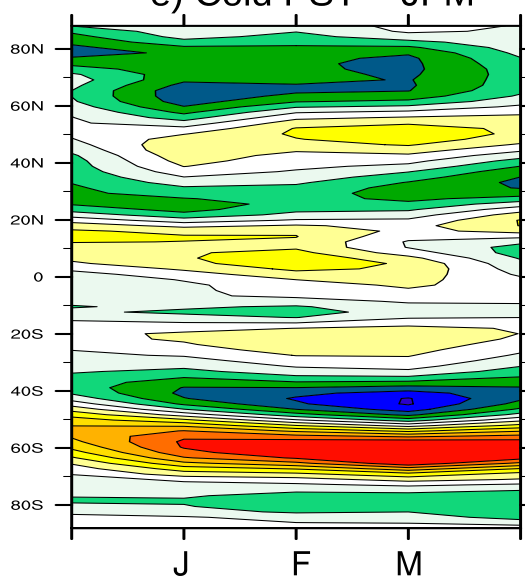

c) High AA - JFM

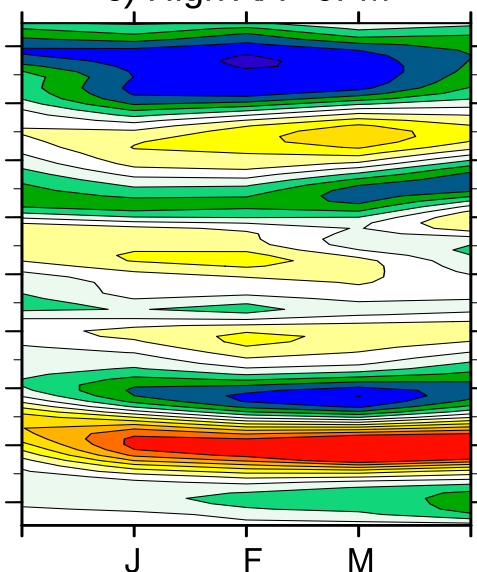

f) Warm PST - JFM

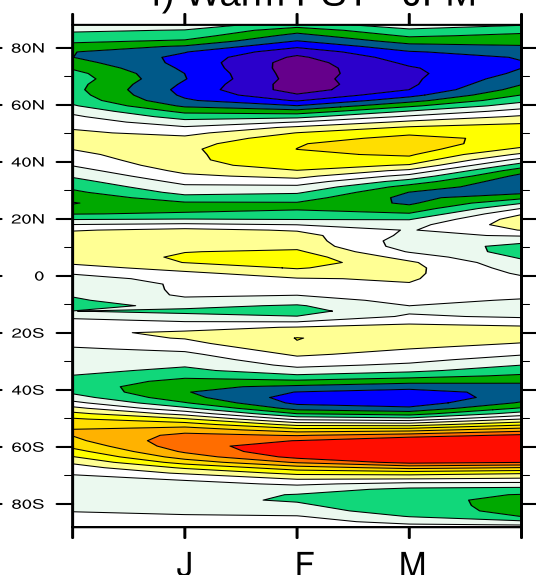

d) High vs Low AA - JFM

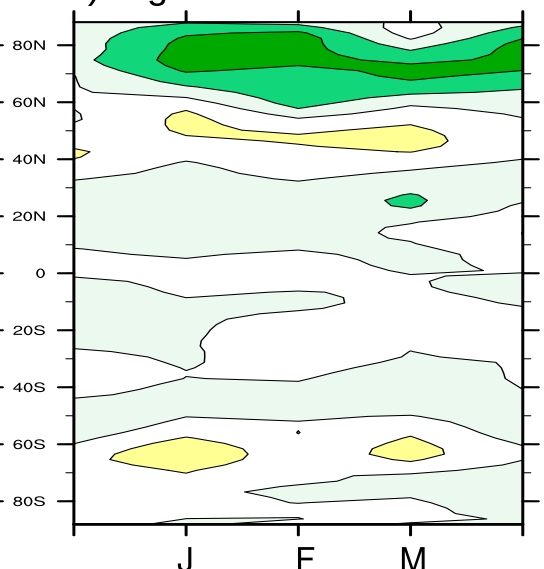

g) Warm vs Cold PST - JFM

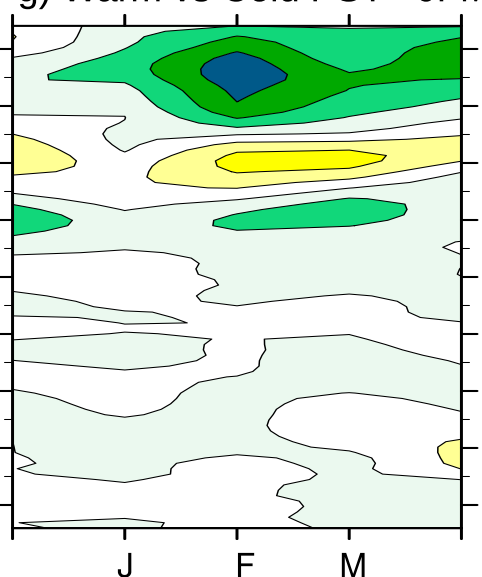

FIG. 8. (a) The 40-member ensemble mean of annual cycle of the 700-hPa zonal wind changes between 2071-2100 and 1981-2010 (color shading; $\mathrm{m} \mathrm{s}^{-1}$ ). Contours indicate the 1981-2010 climatology with a contour interval of $3 \mathrm{~m} \mathrm{~s}^{-1}$. (b) As in (a), but for the 10 members with the lowest AA (small polar surface warming) with only winter shown. (c) As in (b), but for the 10 members with the highest AA (large polar surface warming). (d) Difference between (c) and (b) (high vs low AA). (e)-(g) As in (b)-(d) but for warm vs cold PST. 
with a strong Arctic amplification signal and a decrease in the equator-to-pole temperature gradient at the surface. However, at $50^{\circ} \mathrm{N}$ this does not result in changes in the midlatitude atmospheric dynamics as suggested by the FV12 mechanism. In winter, only the North American sector exhibits a slowdown of the zonal flow and an increase in sinuosity/blocking, as described in Vavrus et al. (2017). Other sectors either exhibit neutral or opposite anomalies, in particular the North Atlantic and North Pacific sectors. The overall decrease in the flow waviness is consistent with results from CMIP5 simulations (Barnes and Polvani 2015; Cattiaux et al. 2016). In summer the response is more consistent with the FV12 mechanism, with negative zonal index anomalies and an increase in sinuosity/blocking on average over the NH. But again, results are strongly sector dependent [supporting conclusions from Overland et al. (2015)] and most of this signal arises from the Asian sector. Associated changes in extreme temperature events do not directly reflect changes in the dynamics since they are mostly driven by thermodynamical changes, in particular Arctic amplification (supporting Ayarzagüena and Screen 2016). When future extreme events are defined relative to the late twenty-first-century temperature distribution (i.e., 10th-90th percentile of the 2071-2100 period), CESM-LENS projects less intense future cold spells in winter over central Europe (but with little modification of their duration and frequency) and slightly more frequent future warm spells in summer over western Europe and Asia (consistent with an increase in variability of the circulation in the AS sector).

CESM-LENS allows us to estimate uncertainties due to internal variability, which is found to be generally very large in terms of changes in midlatitude metrics. The intermember spread of changes in ZON, SIN, and BLO is consistent with the FV12 mechanism, in that $\mathrm{ZON}-$ is associated with $\mathrm{BLO}+$ and $\mathrm{SIN}+$ (i.e., a weaker flow is associated with more waviness and blocking). Even though mean changes do not follow the FV12 mechanism (except over North America), the FV12 mechanism explains a substantial percentage of the intermember spread. A more thorough investigation of the relationships between the metrics and zonal mean temperature change reveals that a larger AA results in a weaker and wavier westerly flow over the Pacific/North America sectors (following the FV12 mechanism). In contrast, the Atlantic sector shows less dependence to $\mathrm{AA}$ and stronger ties to the polar stratospheric temperature. This connection between the zonal index over the North Atlantic sector (i.e., the NAO) and the stratospheric polar vortex is also found in CMIP5 models, adding credence to this finding (Manzini et al. 2014; Cattiaux et al. 2016). Further studies will be necessary to understand whether changes in the lower stratosphere modulate the midlatitude circulation in the troposphere or whether they are forced by changes in the midlatitude flow and in the NAO (Hitchcock and Simpson 2014; Kidston et al. 2015).

The influence of UTW on the intermember spread is hard to discern in CESM-LENS owing to its small intermember variance, but it is clear in the ensemble mean of zonal wind anomalies. The upper-tropospheric warming in the tropics pushes the midlatitude jet poleward, as clearly seen in the SH. In the $\mathrm{NH}$, the Arctic warming pushes the jet in the opposite direction in winter when AA is the most pronounced. Again, this mechanism is consistent with results from CMIP5 simulations (Harvey et al. 2013; Manzini et al. 2014; Deser et al. 2015; Cattiaux et al. 2016). How soon one effect will overwhelm the other is a key question for predicting climate change in midlatitudes on different time scales. Observed trends suggest that the effect of AA has predominated in recent decades (Francis and Vavrus 2015; Cattiaux et al. 2016), although this result is uncertain owing to large internal climate variability over the short period of available observations. In contrast, previous analyses of CMIP5 simulations suggest that future changes in the midlatitude atmospheric circulation will not be primarily driven by AA (Barnes and Polvani 2015; Deser et al. 2015). Rather than demonstrating that one effect dominates the other, our study suggests that both are effective in winter at the end of the twenty-first century, resulting in a narrowing, rather than a latitudinal shift, of the polar front jet stream. As a consequence, the waviness of the midlatitude flow decreases since it is confined to a narrower band of latitudes. Our interpretation reconciles the FV12 mechanism with the late twenty-first-century changes identified in CESM-LENS. The FV12 mechanism acts on one side of the midlatitude flow in winter, but the zonal mean flow responds to both tropical and polar changes (with large variations between sectors). Such a narrowing of the jet under large anthropogenic emissions has also been identified in RCP8.5 simulations of CMIP5 models, which on average simulate a narrowing of midlatitude westerlies and a decrease in sinuosity in winter (Cattiaux et al. 2016, their Figs. $3 b$ and $4 b)$. Therefore, this signal seems to be quite a robust feature in state-of-the-art GCMs forced under the RCP8.5 scenario.

Our study illustrates the complexity of predicting the response of the midlatitude atmospheric dynamics under climate change. A general picture of expected changes cannot be presented since the midlatitude circulation response will depend on the amplitude of several regional processes, in the tropics, at the pole, and in the stratosphere. Uncertainties due to internal 
variability are large, advocating for the necessity of performing large ensemble of simulations with different GCMs when trying to project midlatitude climate change. Moreover, local processes will have to be better identified and understood since the dynamical changes have a large longitudinal asymmetry. Progress on these different aspects will hopefully refine our projections of extreme events and of their societal impact in the midlatitudes in the near and distant future.

Acknowledgments. Thanks are due to Clara Deser, Jennifer Kay, Adam Phillips, the CESM Large Ensemble Community Project, and supercomputing resources provided by NSF/CISL/Yellowstone for making the CESM-LENS data available. YP and GM are supported by NSF Grant AGS-1407360. We acknowledge three anonymous reviewers for their constructive comments that have helped us to improve the manuscript.

\section{REFERENCES}

Andrews, T., J. M. Gregory, M. J. Webb, and K. E. Taylor, 2012: Forcing, feedbacks and climate sensitivity in CMIP5 coupled atmosphere-ocean climate models. Geophys. Res. Lett., 39, L09712, doi:10.1029/2012GL051607.

Ayarzagüena, B., and J. A. Screen, 2016: Future Arctic sea ice loss reduces severity of cold air outbreaks in midlatitudes. Geophys. Res. Lett., 43, 2801-2809, doi:10.1002/2016GL068092.

Barnes, E. A., 2013: Revisiting the evidence linking Arctic amplification to extreme weather in midlatitudes. Geophys. Res. Lett., 40, 4734-4739, doi:10.1002/grl.50880.

— , and L. M. Polvani, 2015: CMIP5 projections of Arctic amplification, of the North American/North Atlantic circulation, and of their relationship. J. Climate, 28, 5254-5271, doi:10.1175/JCLI-D-14-00589.1.

__ , and J. A. Screen, 2015: The impact of Arctic warming on the midlatitude jetstream: Can it? Has it? Will it? Wiley Interdiscip. Rev.: Climate Change, 6, 277-286, doi:10.1002/ wcc. 337 .

Blackport, R., and P. J. Kushner, 2016: The transient and equilibrium climate response to rapid summertime sea ice loss in CCSM4. J. Climate, 29, 401-417, doi:10.1175/JCLI-D-15-0284.1.

Butler, A. H., D. W. Thompson, and R. Heikes, 2010: The steadystate atmospheric circulation response to climate change-like thermal forcings in a simple general circulation model. J. Climate, 23, 3474-3496, doi:10.1175/2010JCLI3228.1.

Cattiaux, J., and C. Cassou, 2013: Opposite CMIP3/CMIP5 trends in the wintertime Northern Annular Mode explained by combined local sea ice and remote tropical influences. Geophys. Res. Lett., 40, 3682-3687, doi:10.1002/grl.50643.

— , Y. Peings, D. Saint-Martin, N. Trou-Kechout, and S. Vavrus, 2016: Sinuosity of midlatitude atmospheric flow in a warming world. Geophys. Res. Lett., 43, 8259-8268, doi:10.1002/ 2016GL070309.

Chen, G., J. Lu, D. A. Burrows, and L. R. Leung, 2015: Local finiteamplitude wave activity as an objective diagnostic of midlatitude extreme weather. Geophys. Res. Lett., 42, 10952-10960, doi:10.1002/2015GL066959.
Cohen, J., and Coauthors, 2014: Recent Arctic amplification and extreme mid-latitude weather. Nat. Geosci., 7, 627-637, doi:10.1038/ngeo2234.

Deser, C., A. S. Phillips, M. A. Alexander, and B. V. Smoliak, 2014: Projecting North American climate over the next 50 years: Uncertainty due to internal variability. J. Climate, 27, 22712296, doi:10.1175/JCLI-D-13-00451.1.

_ R. A. Tomas, and L. Sun, 2015: The role of ocean-atmosphere coupling in the zonal-mean atmospheric response to Arctic sea ice loss. J. Climate, 28, 2168-2186, doi:10.1175/JCLI-D-14-00325.1.

$\longrightarrow$, L. Sun, R. A. Tomas, and J. Screen, 2016: Does ocean coupling matter for the northern extratropical response to projected Arctic sea ice loss? Geophys. Res. Lett., 42, 2149-2157, doi:10.1002/2016GL067792.

Francis, J. A., and S. J. Vavrus, 2012: Evidence linking Arctic amplification to extreme weather in mid-latitudes. Geophys. Res. Lett., 39, L06801, doi:10.1029/2012GL051000.

_ and _ 2015: Evidence for a wavier jet stream in response to rapid Arctic warming. Environ. Res. Lett., 10, 014005, doi:10.1088/1748-9326/10/1/014005.

Hall, R., R. Erdélyi, E. Hanna, J. M. Jones, and A. A. Scaife, 2014: Drivers of North Atlantic Polar Front jet stream variability. Int. J. Climatol., 35, 1697-1720, doi:10.1002/joc.4121.

Harvey, B. J., L. C. Shaffrey, and T. J. Woollings, 2013: Equator-topole temperature differences and the extra-tropical storm track responses of the CMIP5 climate models. Climate Dyn., 43, 1171-1182, doi:10.1007/s00382-013-1883-9.

Hassanzadeh, P., and Z. Kuang, 2015: Blocking variability: Arctic amplification versus Arctic Oscillation. Geophys. Res. Lett., 42, 8586-8595, doi:10.1002/2015GL065923.

,-- , and B. F. Farrell, 2014: Responses of midlatitude blocks and wave amplitude to changes in the meridional temperature gradient in an idealized dry GCM. Geophys. Res. Lett., 41, 5223-5232, doi:10.1002/2014GL060764.

Held, I. M., 1993: Large-scale dynamics and global warming. Bull. Amer. Meteor. Soc., 74, 228-241, doi:10.1175/ 1520-0477(1993)074<0228:LSDAGW>2.0.CO;2.

Hitchcock, P., and I. R. Simpson, 2014: The downward influence of stratospheric sudden warmings. J. Atmos. Sci., 71, 3856-3876, doi:10.1175/JAS-D-14-0012.1.

Holland, M. M., and C. M. Bitz, 2003: Polar amplification of climate change in coupled models. Climate Dyn., 21, 221-232, doi:10.1007/s00382-003-0332-6.

Hurrell, J., and W. H. van Loon, 1997: Decadal variations in climate associated with the North Atlantic Oscillation. Climatic Change, 36, 301-326, doi:10.1023/A:1005314315270.

— , and Coauthors, 2013: The Community Earth System Model: A framework for collaborative research. Bull. Amer. Meteor. Soc., 94, 1339-1360, doi:10.1175/BAMS-D-12-00121.1.

Kay, J. E., M. M. Holland, C. Bitz, A. Gettelman, E. BlanchardWrigglesworth, A. Conley, and D. Bailey, 2012: The influence of local feedbacks and northward heat transport on the equilibrium Arctic climate response to increased greenhouse gas forcing. J. Climate, 25, 5433-5450, doi:10.1175/JCLI-D-11-00622.1.

— , and Coauthors, 2015: The Community Earth System Model (CESM) large ensemble project: A community resource for studying climate change in the presence of internal climate variability. Bull. Amer. Meteor. Soc., 96, 1333-1349, doi:10.1175/ BAMS-D-13-00255.1.

Kennedy, D., T. Parker, T. Woollings, B. Harvey, and L. Shaffrey, 2016: The response of high-impact blocking weather systems to climate change. Geophys. Res. Lett., 43, 7250-7258, doi:10.1002/ 2016GL069725. 
Kidston, J., A. A. Scaife, S. C. Hardiman, D. M. Mitchell, N. Butchart, M. P. Baldwin, and L. L. Gray, 2015: Stratospheric influence on tropospheric jet streams, storm tracks and surface weather. Nat. Geosci., 8, 433-440, doi:10.1038/ngeo2424.

Kim, B.-M., S.-W. Son, S.-K. Min, J.-H. Jeong, S.-J. Kim, X. Zhang, T. Shim, and J.-H. Yoon, 2014: Weakening of the stratospheric polar vortex by Arctic sea-ice loss. Nat. Commun., 5, 4646, doi:10.1038/ncomms5646.

Liu, J., J. A. Curry, H. Wang, M. Song, and M. R. Horton, 2012: Impact of declining Arctic sea ice on winter snowfall. Proc. Natl. Acad. Sci. USA, 109, 4074-4079, doi:10.1073/pnas.1114910109.

Manabe, S., and R. T. Wetherald, 1975: The effects of doubling the $\mathrm{CO}_{2}$ concentration on the climate of a general circulation model. J. Atmos. Sci., 32, 3-15, doi:10.1175/ 1520-0469(1975)032<0003:TEODTC > 2.0.CO;2.

Manzini, E., and Coauthors, 2014: Northern winter climate change: Assessment of uncertainty in CMIP5 projections related to stratosphere-troposphere coupling. J. Geophys. Res. Atmos., 119, 7979-7998, doi:10.1002/2013JD021403.

Overland, J. E., J. Francis, R. Hall, E. Hanna, S.-J. Kim, and T. Vihma, 2015: The melting Arctic and midlatitude weather patterns: Are they connected? J. Climate, 28, 7917-7932, doi:10.1175/JCLI-D-14-00822.1.

Peings, Y., and G. Magnusdottir, 2014: Response of the wintertime Northern Hemisphere atmospheric circulation to current and projected Arctic sea ice decline: A numerical study with CAM5. J. Climate, 27, 244-264, doi:10.1175/JCLI-D-13-00272.1.

Perlwitz, J., M. Hoerling, and R. Dole, 2015: Arctic tropospheric warming causes and linkages to lower latitudes. J. Climate, 28, 2154-2167, doi:10.1175/JCLI-D-14-00095.1.

Schneider, T., T. Bischoff, and H. Płotka, 2015: Physics of changes in synoptic midlatitude temperature variability. J. Climate, $\mathbf{2 8}$, 2312-2331, doi:10.1175/JCLI-D-14-00632.1.

Screen, J. A., 2013: Influence of Arctic sea ice on European summer precipitation. Environ. Res. Lett., 8, 044015, doi:10.1088/ 1748-9326/8/4/044015.

_ 2014: Arctic amplification decreases temperature variance in northern mid- to high-latitudes. Nat. Climate Change, 4, 577582, doi:10.1038/nclimate2268.

_ , and I. Simmonds, 2010: The central role of diminishing sea ice in recent Arctic temperature amplification. Nature, 464, 13341337, doi:10.1038/nature09051.
— and _ 2013: Exploring links between Arctic amplification and mid-latitude weather. Geophys. Res. Lett., 40, 959-964, doi:10.1002/grl.50174.

— C. Deser, and L. Sun, 2015: Projected changes in regional climate extremes arising from Arctic sea ice loss. Environ. Res. Lett., 10, 084006, doi:10.1088/1748-9326/10/8/084006.

Simpson, I. R., T. A. Shaw, and R. Seager, 2014: A diagnosis of the seasonally and longitudinally varying mid-latitude circulation response to global warming. J. Atmos. Sci., 71, 2489-2515, doi:10.1175/JAS-D-13-0325.1.

Smith, K. L., and L. M. Polvani, 2014: The surface impacts of Arctic stratospheric ozone anomalies. Environ. Res. Lett., 9, 074015, doi:10.1088/1748-9326/9/7/074015.

Taylor, K. E., R. J. Stouffer, and G. A. Meehl, 2012: The CMIP5 experiment design. Bull. Amer. Meteor. Soc., 93, 485-498, doi:10.1175/BAMS-D-11-00094.1.

Thompson, D. W. J., and J. M. Wallace, 1998: The Arctic Oscillation signature in the wintertime geopotential height and temperature fields. Geophys. Res. Lett., 25, 1297-1300, doi:10.1029/98GL00950.

Tibaldi, S., and F. Molteni, 1990: On the operational predictability of blocking. Tellus, 42A, 343-365, doi:10.3402/tellusa.v42i3.11882.

Vavrus, S., F. Wang, J. E. Martin, J. A. Francis, Y. Peings, and J. Cattiaux, 2017: Changes in North American atmospheric circulation and extreme weather: Influence of Arctic amplification and Northern Hemisphere snow cover. J. Climate, 30, 4317-4333, doi:10.1175/JCLI-D-16-0762.1.

Vihma, T., 2014: Effects of Arctic Sea Ice Decline on Weather and Climate: A Review. Surv. Geophys., 35, 1175-1214, doi:10.1007/ s10712-014-9284-0.

Wallace, J. M., I. M. Held, D. W. J. Thompson, K. E. Trenberth, and J. E. Walsh, 2014: Global warming and winter weather. Science, 343, 729-730, doi:10.1126/science.343.6172.729.

Walsh, J. E., 2014: Intensified warming of the Arctic: Causes and impacts on middle latitudes. Global Planet. Change, 117, 5263, doi:10.1016/j.gloplacha.2014.03.003.

Woollings, T., 2008: Vertical structure of anthropogenic zonalmean atmospheric circulation change. Geophys. Res. Lett., $\mathbf{3 5}$, L19702, doi:10.1029/2008GL034883.

Zhang, J., W. S. Tian, M. P. Chipperfield, F. Xie, and J. Huang, 2016: Persistent shift of the Arctic polar vortex towards the Eurasian continent in recent decades. Nat. Climate Change, 6, 1094-1099, doi:10.1038/nclimate3136. 